\title{
Uncoupling of UNC5C with Polymerized TUBB3 in Microtubules Mediates Netrin-1 Repulsion
}

\author{
(1)Qiangqiang Shao, Tao Yang, Huai Huang, Farrah Alarmanazi, and $₫$ Guofa Liu \\ Department of Biological Sciences, University of Toledo, Toledo, Ohio 43606
}

Modulation of microtubule (MT) dynamics is a key event of cytoskeleton remodeling in the growth cone (GC) during axon outgrowth and pathfinding. Our previous studies have shown that the direct interaction of netrin receptor DCC and DSCAM with polymerized TUBB3, a neuron-specific MT subunit in the brain, is required for netrin-1-mediated axon outgrowth, branching, and attraction. Here, we show that uncoupling of polymerized TUBB3 with netrin-1-repulsive receptor UNC5C is involved in netrin-1-mediated axonal repulsion. TUBB3 directly interacted with UNC5C and partially colocalized with UNC5C in the peripheral area of the GC of primary neurons from the cerebellar external granule layer of P2 mouse pups of both sexes. Netrin-1 reduced this interaction as well as the colocalization of UNC5C and TUBB 3 in the GC. Results from the in vitro cosedimentation assay indicated that UNC5C interacted with polymerized TUBB3 in MTs and netrin-1 decreased this interaction. Knockdown of either TUBB3 or UNC5C blocked netrin-1-promoted axon repulsion in vitro and caused defects in axon projection of DRG toward the spinal cord in vivo. Furthermore, live-cell imaging of end-binding protein 3 tagged with EGFP (EB3-GFP) in primary external granule layer cells showed that netrin-1 differentially increased MT dynamics in the GC with more MT growth in the distal than the proximal region of the GC during repulsion, and knockdown of either UNC5C or TUBB3 abolished the netrin-1 effect. Together, these data indicate that the disengagement of UNC5C with polymerized TUBB3 plays an essential role in netrin-1/UNC5C-mediated axon repulsion.

Key words: axon guidance; growth cone; microtubule dynamics; netrin; TUBB3; UNC5C

\section{Significance Statement}

Proper regulation of microtubule (MT) dynamics in the growth cone plays an important role in axon guidance. However, whether guidance cues modulate MT dynamics directly or indirectly is unclear. Here, we report that dissociation of UNC5C and polymerized TUBB3, the highly dynamic $\beta$-tubulin isoform in neurons, is essential for netrin-1/UNC5C-promoted axon repulsion. These results not only provide a working model of direct modulation of MTs by guidance cues in growth cone navigation but also help us to understand molecular mechanisms underlying developmental brain disorders associated with TUBB3 mutations.

\section{Introduction}

In the developing nervous system, growth cone (GC) navigation is guided by extracellular signals, such as guidance cues, growth factors, and cell adhesion molecules (Guan and Rao, 2003; Dent et al., 2011; Kolodkin and Tessier-Lavigne, 2011). Both actin and microtubule (MT) dynamics in the GC are essential for directing axon projection and pathfinding (Buck and Zheng, 2002; Dent

\footnotetext{
Received Aug. 17, 2016; revised April 22, 2017; accepted April 28, 2017.

Author contributions: G.L. designed research; Q.S., T.Y., and H.H. performed research; Q.S. and F.A. analyzed data; Q.S. and G.L. wrote the paper.

This work was supported by the Whitehall Foundation and National Institutes of Health. We thank Dr. David L. Turner for the TUBB3 shRNA constructs; Dr. Niels Galjart for EB3-GFP; and Dr. Elizabeth C. Engle for TUBB3 constructs. The authors declare no competing financial interests.

Correspondence should be addressed to Dr. Guofa Liu, Department of Biological Sciences, University of Toledo, 2801 West Bancroft Street, Toledo, OH 43606. E-mail: Guofa.Liu@utoledo.edu.

DOI:10.1523/JNEUROSCI.2617-16.2017

Copyright $\odot 2017$ the authors $\quad 0270-6474 / 17 / 375620-14 \$ 15.00 / 0$
}

and Gertler, 2003; Kalil and Dent, 2005; Lowery and Van Vactor, 2009; Dent et al., 2011; Vitriol and Zheng, 2012; Liu and Dwyer, 2014). In addition to consolidating and providing mechanical support to GC steering initiated by actin dynamics, MTs also play an essential and instructive role for GC behavior (Tanaka and Kirschner, 1995; Challacombe et al., 1997; Buck and Zheng, 2002; Dent et al., 2004, 2011; Qu et al., 2013a; Huang et al., 2015). However, the molecular mechanisms linking guidance signaling to MT dynamics remain unclear.

Netrin-1 is a classical guidance cue involved in axon guidance and neuronal migration in the developing nervous system (Tessier-Lavigne et al., 1988; Hedgecock et al., 1990; Ishii et al., 1992; Kennedy et al., 1994; Kolodziej et al., 1996). As a bifunctional guidance cue, netrin-1 is capable of attracting or repelling axon projection depending on assembling of appropriate receptor complexes (Colamarino and Tessier-Lavigne, 1995; Alcántara et al., 2000; Quinn and Wadsworth, 2008; Lai Wing Sun et al., 
2011; Liu and Dwyer, 2014). For instance, collaboration of Deleted in Colorectal Cancer (DCC) with Down Syndrome Adhesion Molecule (DSCAM) mediates netrin-1-induced axon outgrowth, branching, and attraction (Keino-Masu et al., 1996; Fazeli et al., 1997; Ly et al., 2008; Liu et al., 2009; Huang et al., 2015), although DSCAM knock-out mice do not exhibit guidance defects of spinal cord commissural axon projections (Palmesino et al., 2012). In contrast, Uncoordinated-5 (UNC5), alone or interaction with either DCC or DSCAM, is involved in axon repulsion (Keino-Masu et al., 1996; Kolodziej et al., 1996; Ackerman et al., 1997; Leonardo et al., 1997; Hong et al., 1999; Finger et al., 2002; Purohit et al., 2012). Our recent studies have shown that TUBB3, the highly dynamic $\beta$-tubulin isoform in neurons, interacts directly with DCC and DSCAM (Qu et al., 2013a; Huang et al., 2015). Netrin-1 induces the interaction of polymerized TUBB3 with DCC and DSCAM. Furthermore, TUBB3 is required for netrin-1-induced axon outgrowth, branching, and attraction in the developing nervous system (Qu et al., 2013a; Huang et al., 2015). These results suggest that the interaction of TUBB3 with DCC and DSCAM directly couples netrin-1 signaling to MT dynamics in axon outgrowth and attraction.

Here we demonstrate that the repulsive netrin-1 receptor UNC5C also interacts with TUBB3. Netrin-1 decreases the interaction of UNC5C with polymerized TUBB3 in primary neurons. Both UNC5C and TUBB3 are involved in netrin-1-induced differential MT growth in the GC during axon repulsion. Moreover, TUBB3 is required for netrin-1/UNC5C-mediated axon repulsion in vitro and DRG axon projection in vivo. These results lead to a working model in which disengagement of UNC5C with dynamic TUBB3 in MTs plays an essential role in axon repulsion induced by netrin-1.

\section{Materials and Methods}

Materials. The following antibodies were used: rabbit anti-FLAG (Abcam catalog \#ab124462, RRID:AB_11000959), rabbit anti-UNC5C (Abcam catalog \#ab89938, RRID:AB_2050439), rabbit anti-TUBB2 (Abcam catalog \#ab80326, RRID:AB_1604033), rabbit anti-His (Abcam catalog \#ab14923, RRID:AB_443105), rabbit anti-TUBB3 (Abcam catalog \#ab2276-1, RRID:AB_1267370), rabbit anti-hemagglutinin (HA) (Santa Cruz Biotechnology catalog \#sc-805, RRID:AB_631618), mouse antiTUBB3 (Covance catalog \#MMS-435P, RRID:AB_2313773), mouse anti-Myc (Calbiochem catalog \#OP10F-100UG, RRID:AB_564474), rabbit anti-TUBB1 (Abiocode catalog \#R0742-vp, RRID:AB_2631111), mouse BEN antibody (DSHB, RRID:AB_2313998), rabbit anti-GST (Cell Signaling Technology catalog \#2622, RRID:AB_331670), bovine anti-mouse IgG-HRP (Santa Cruz Biotechnology catalog \#sc-2371, RRID:AB_634824), goat anti-rabbit IgG-HRP (Santa Cruz Biotechnology catalog \#sc-2004, RRID:AB_631746), donkey anti-goat IgG-HRP (Santa Cruz Biotechnology catalog \#sc-2020, RRID:AB_631728), AlexaFluor488 goat anti-mouse IgG (Invitrogen catalog \#A-21121, RRID:AB_141514), and AlexaFluor-647 goat anti-rabbit IgG (Invitrogen catalog \#A-21244, RRID:AB_141663).

Taxol and nocodazole were obtained from MP Biochemicals. Purified TUBB3 was purchased from Atgen. The cDNA encoding intracellular and transmembrane domains of human UNC5C (UNC5C-ICD) tagged with GST were subcloned into p-Dest 565 vectors via Gateway technology (Invitrogen), and recombinant UNC5C-ICD was purified from BL21 competent Escherichia coli. UNC5C control shRNA, UNC5C shRNA, full-length human UNC5C-HA, TUBB3-FLAG, and Venus-YFP have been described previously (Purohit et al., 2012; Qu et al., 2013a). EB3-GFP constructs were gifts from Niels Galjart. TUBB3-FLAG was modified from TUBB3-V5, a gift from Elizabeth C. Engle. TUBB3 shRNA and control shRNA are from Dr. David L. Turner (Yu et al., 2002). Netrin-1 was either obtained from R\&D Systems or purified with anti-Myc tag affinity matrix from the conditioned media of HEK cells stably secreting netrin- 1 . The control was made by sham purification from the conditioned media from HEK cells without expression of Myc-tagged netrin-1.

Primary neuron cultures. Cerebellar neuron cultures were prepared from postnatal mouse pups of either sex as previously described (Liu et al., 2004, 2007, 2009; Purohit et al., 2012). For nucleofection, dissociated neurons from postnatal day 4 (P4) mouse cerebella or P2 external granule layer (EGL) were mixed with specific constructs and nucleofected (Amaxa) using program G-013 (Purohit et al., 2012). For MT cosedimentation assay, transfected cerebellar neurons were cultured on polyL-lysine (PLL, $200 \mu \mathrm{g} / \mathrm{ml}$ )-coated Petri dishes for $2 \mathrm{~d}$ and cell lysates then analyzed by immunoblotting. For the Dunn chamber axon guidance assay, P2 cerebellar EGL neurons after nucleofection were plated onto PLL-coated coverslips and cultured in DMEM + B27 + $20 \mathrm{U} / \mathrm{ml}$ of penicillin/streptomycin at $37^{\circ} \mathrm{C}$ with $5 \% \mathrm{CO}_{2}$ for $2-3 \mathrm{~d}$.

Immunoprecipitation and immunoblotting. HeLa cells were transfected with the PEI method and cultured $24 \mathrm{~h}$ after transfection. P4 mouse cerebellar neurons were dissociated, transfected, and cultured as previously described (Purohit et al., 2012). For netrin-1 stimulation, transfected HeLa cells and primary neurons were starved for $6 \mathrm{~h}$ in serum-free DMEM media and followed by incubation with either netrin-1-conditioned media or purified netrin-1 protein $(200 \mathrm{ng} / \mathrm{ml})$ up to $20 \mathrm{~min}$. The control was made by either conditioned media from regular HEK293 cells that had not been transfected with a cDNA expressing the Myc-tagged netrin-1 or sham purification from the conditioned media from HEK cells. Primary neurons or HeLa cells were lysed and cell lysates incubated with specific antibodies and protein A/G-agarose beads (Santa Cruz Biotechnology) overnight at $4^{\circ} \mathrm{C}$, as described previously (Purohit et al., 2012). Protein extracts were separated with 7.5\% SDS-PAGE and immunoblotted with specific antibodies. Western blots were visualized with the enhanced chemiluminescence kit (Fisher).

MT cosedimentation assay. Primary cerebellar neurons from P4 mice were cultured with culture medium (DMEM $+10 \% \mathrm{FBS}+20 \mathrm{U} / \mathrm{ml}$ of penicillin/streptomycin) for $16 \mathrm{~h}$ and stimulated by purified netrin-1 (200 ng/ml) or sham-purified control for $5 \mathrm{~min}$ (Liu et al., 2004, 2007, 2008, 2009; Purohit et al., 2012; Qu et al., 2013a; Huang et al., 2015). Primary neurons were lysed in the RIPA buffer $(25 \mathrm{~mm}$ Tris, $150 \mathrm{~mm}$ $\mathrm{NaCl}, 0.1 \%$ SDS, 5 mm EDTA, 0.5\% sodium deoxycholate, 1\% NP-40, protease inhibitor mixture, $\mathrm{pH} 8$ ) and cell lysates centrifuged at $100,000 \times g$ for $1 \mathrm{~h}$ at $4^{\circ} \mathrm{C}$. To stabilize MTs, the supernatant was incubated with $40 \mu \mathrm{M}$ Taxol or DMSO in PEMG buffer (100 mM PIPES, $1 \mathrm{~mm}$ EGTA, $1 \mathrm{~mm} \mathrm{MgSO}_{4}, 1 \mathrm{~mm}$ GTP, pH 6.8) at room temperature for 30 min, and MTs were pelleted by centrifugation through a $10 \%$ sucrose cushion at $50,000 \times g$ for $30 \mathrm{~min}$ at $25^{\circ} \mathrm{C}$ as previously described ( $\mathrm{Qu}$ et al., 2013a; Huang et al., 2015). After resuspension of pellet in tubulin buffer (50 mм HEPES, 1 mм MgCl 2 , 1 mм EGTA, 10\% glycerol, 150 mм $\mathrm{KCl}, 40 \mu \mathrm{M}$ taxol, $1 \mathrm{~mm}$ GTP, $5 \mathrm{~mm}$ Mg-ATP, $1 \mathrm{~mm}$ PMSF, $1 \times$ protease inhibitor mixture), proteins in the supernatant and the pellet were analyzed by Western blotting.

Immunofluorescence. Primary EGL cells from P2 mouse cerebella were grown on PLL-coated coverslips and treated with either sham purified control or purified netrin-1 $(200 \mathrm{ng} / \mathrm{ml})$ for 5 or $10 \mathrm{~min}$. Neurons were fixed in prewarmed $4 \% \mathrm{PFA}$ in DMEM at $37^{\circ} \mathrm{C}$ for $30 \mathrm{~min}$ and permeabilized with PBST (0.5\% Triton X-100 in PBS) for $15 \mathrm{~min}$. Cells were blocked with $0.25 \% \mathrm{BSA}+0.1 \%$ Triton in PBS at room temperature for $30 \mathrm{~min}$ and then incubated with primary antibody solution containing rabbit anti-UNC5C and mouse anti-TUBB3 antibodies overnight at $4^{\circ} \mathrm{C}$. Neurons were incubated with fluorescent secondary antibodies (AlexaFluor-488 donkey anti-mouse IgG and AlexaFluor-647 donkey anti-rabbit IgG), and images of growth cones were taken sequentially using a confocal microscope (Leica Microsystems, TCS, SP8). Fluorescent signals were acquired in a photon counting mode using Leica Microsystems HyD detector with the same setting, and the Pearson correlation coefficient (PCC) of each ROI (the peripheral region of GCs, including lamellipodia and filopodia) was calculated using the colocalization module of Leica Microsystems confocal software (RRID:SCR_013673). PCC values from different groups were analyzed with a one-way ANOVA with Tukey's test for post hoc comparisons. 
Axon projection of chicken DRG neurons in vivo. Fertilized White Leghorn chicken eggs were incubated and embryonic development was staged as described previously (Liu et al., 2004, 2007, 2009; Qu et al., 2013a, b; Huang et al., 2015). At Stages 12-16, either Venus YFP only or Venus YFP plus specific plasmids or siRNAs were injected into the neural tube of chicken embryos, and the in ovo electroporation was performed with the following program: $25 \mathrm{~V}, 5 \mathrm{~ms}, 5$ pulses (BTX ECM830) (Liu et al., 2004, 2007, 2009; Qu et al., 2013a, b; Huang et al., 2015). At Stages 23-25, chicken embryos were collected and lumbosacral segments of the chick spinal cords labeled with YFP fluorescence were isolated under a fluorescent microscope. Transverse $200 \mu \mathrm{m}$ sections of chicken spinal cords were prepared, fixed in 4\% PFA in $1 \times$ PBS, permeabilized with PBST (1\% Triton X-100 in $1 \times$ PBS), and stained with BEN antibody (SC1/DM-GRASP protein, 1:10 in $1 \times$ PBS). The fluorescence images of DRG axons obtained under a confocal microscope (Leica Microsystems, TCS, SP8) and the size of the dorsal root entry zone (DREZ) were measured using the Imaging software (Leica Microsystems, Application Suite $\mathrm{X})$, Data were analyzed with a one-way ANOVA with Tukey's test for post hoc comparisons (Masuda et al., 2008).

Dunn chamber axon guidance assay. P2 cerebellar EGL neurons were nucleofected and grown on PLL-coated square coverslips (Erie Scientific). Dunn chambers were assembled as previously described (Yam et al., 2009) with some modifications. Briefly, the Dunn chamber was rinsed by DMEM once and then twice with culture media (DMEM + B27 + 20 U/ml of penicillin/streptomycin) before assembly. The inner and outer wells were filled with culture medium, and a coverslip with neurons was then inverted over the Dunn chamber, leaving a narrow slit at the edge of one side. Other three sides of the coverslip were sealed with Petroleum Jelly (VL-JON Laboratory), and media in outer well was drained and replaced by culture media containing either sham-purified control or purified netrin-1 (200 ng/ml). The narrow slit was then sealed with Petroleum Jelly. The assembly process was completed within 5 min. After Dunn chamber assembly, time-lapse videos of YFP-labeled EGL neurons in the bridge region of the Dunn chamber were acquired every $5 \mathrm{~min}$ for $90 \mathrm{~min}$ using a confocal microscope (Leica Microsystems, TCS SP8). Most GCs of EGL neurons showed significant repulsion within 40-55 $\mathrm{min}$ in response to a netrin-1 gradient, which was defined as "the turning stage." Axon turning (angle turned) was evaluated by the angle between initial and final angel of an axon as described previously (Yam et al., 2009).

Time-lapse recording of EB3-GFP in primary cerebellar EGL neurons. P2 cerebellar EGL neurons were nucloefected with EB3-GFP plus specific plasmids or siRNAs and cultured for $48 \mathrm{~h}$ before live imaging. The Dunn chamber axon guidance assay was performed in a $37^{\circ} \mathrm{C}$ chamber using a confocal microscope (Leica Microsystems, TCS, SP8). Images were taken every 1-3.5 s over 60 frames per GC and corrected for photobleaching and background subtraction using Fiji. Images were passed through a bandpass filter before particle detection and trajectories of EB3-GFP comets in the GC were tracked using the Fiji (RRID:SCR_002285) with MTrackJ plug-in (http://www.imagescience.org/meijering/software/ mtrackj/). Only comets that could be tracked for $>3$ frames were considered moving comets. Movies acquired at 10, 40, and $60 \mathrm{~min}$ were used to analyze EB3-GFP comet behavior in the GC at the preturn, turning, and post-turn stages, respectively.

\section{Results}

UNC5C interacts with TUBB3 in a netrin-1-dependent manner TUBB3 couples netrin-1/DCC signaling to MT dynamics in axon attraction (Qu et al., 2013a; Liu and Dwyer, 2014). To determine whether TUBB3 is also involved in netrin repulsion, we examined the potential interaction of TUBB3 with UNC5C. FLAG-tagged human TUBB3 (TUBB3-FLAG) and UNC5C-HA were transiently expressed in HeLa cells. Cell lysates were immunoprecipitated with anti-HA antibody and analyzed by Western blotting with anti-FLAG and anti-HA antibodies. TUBB3 was readily detected in the lysates immunoprecipitated with anti-HA antibody (UNC5C) (Fig. 1A), suggesting that the two proteins may interact. To determine whether endogenous TUBB3 interacted with UNC5C, we performed coimmunoprecipitation on the lysates of dissociated cerebellar neurons from postnatal day 4 (P4) mice, which strongly express UNC5C and respond to netrin-1 with repulsion (Bartoe et al., 2006; Purohit et al., 2012). Anti-UNC5C antibody coimmunoprecipitated TUBB3 (Fig. 1B, left). The interaction of endogenous UNC5C with TUBB3 was confirmed by the reverse coimmunoprecipitation (Fig. $1 B$, right). Treatment of dissociated neurons with netrin-1 decreased the interaction of UNC5C with TUBB3 within $3 \mathrm{~min}$ and the reduction lasted up to 10 min after netrin-1 stimulation (Fig. $1 B, D$, quantification in $C$ ). The interaction of endogenous UNC5C with TUBB3 in P4 cerebellar neurons was decreased in a dosedependent manner in response to netrin-1 (Fig. 1E). UNC5C did not interact with endogenous TUBB1 and TUBB2, two other $\beta$-tubulin subunits, with or without netrin-1 stimulation (Fig. $1 F$ ). These results strongly suggest that TUBB3 specifically interacts with UNC5C in primary P4 cerebellar neurons. To further determine whether UNC5C directly interacts with TUBB3, a truncated protein containing only the intracellular and transmembrane domains of UNC5C (UNC5C-ICD-GST) was purified and incubated with purified TUBB3. TUBB3 appeared to interact directly with UNC5C-ICD (Fig. 1G).

\section{Netrin-1 regulates colocalization of UNC5C with TUBB3}

UNC5C is enriched in the axon GC of EGL neurons from developing mouse cerebella and mediates netrin-1-promoted GC collapse and repulsion (Bartoe et al., 2006; Purohit et al., 2012). To determine whether endogenous UNC5C colocalizes with TUBB3 in the GC, primary neurons from the P2 mouse EGL were cultured and stimulated with netrin-1. Quantitative colocalization analysis of confocal fluorescence microscopy images showed partial overlap of immunofluorescent signals of UNC5C and TUBB3 in the peripheral region of GCs, including lamellipodia and filopodia (Fig. $2 A-C$, quantification in $J$ ), suggesting that UNC5C partially colocalized with TUBB3 in the GC. Netrin-1 decreased the overlap of UNC5C with TUBB3 in the GC of P2 EGL neurons (Fig. $2 D-I$, quantification in $J$ ): the PPC was reduced from $0.59 \pm$ 0.02 in the control group (sham-purified control) to $0.49 \pm 0.02$ in the 5 min netrin- 1 stimulation group and to $0.37 \pm 0.02$ in the 10 min netrin-1 treatment group. As a control, the PCC approximated zero in each group after one fluorescent channel of the same confocal image was rotated 90 degrees (Fig. $2 \mathrm{~K}$ ), indicating that the red and green signals had a high degree of correlation, not an artificial overlap of random signals (Tcherkezian et al., 2010; Dunn et al., 2011; Horn et al., 2016). These results suggest that netrin- 1 decreases the colocalization of UNC5C with TUBB3 in the GC of primary P2 EGL cells.

\section{Netrin-1 inhibits the interaction of UNC5C with dynamic TUBB3 in MTs}

Heterodimers of $\alpha$ - and $\beta$-tubulin polymerize into dynamic MTs. Our previous studies have shown that dynamic MTs are essential for the interaction of TUBB3 with DCC or DSCAM in axon outgrowth, branching, and attraction ( $\mathrm{Qu}$ et al., 2013a; Huang et al., 2015). To examine the potential role of MT dynamics in the binding of TUBB3 to UNC5C, primary P4 cerebellar neurons were cultured and treated with the MT-stabilizing drug paclitaxel (Taxol) or the MT-destabilizing drug nocodazole to disrupt MT dynamics before coimmunoprecipitation. As we observed previously, UNC5C was coimmunoprecipitated with TUBB3 and netrin-1 decreased this interaction (Fig. $3 A, B$ ). Pretreatment with Taxol or nocodazole abolished the ability of netrin- 1 to reduce the TUBB3/UNC5C interaction (Fig. $3 A, B$ ). Given that both Taxol and nocodazole disrupt MT dynamics, 
A

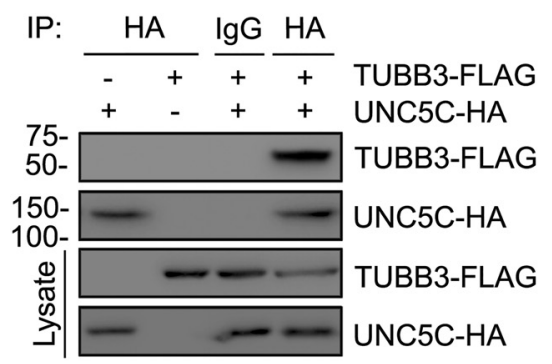

D
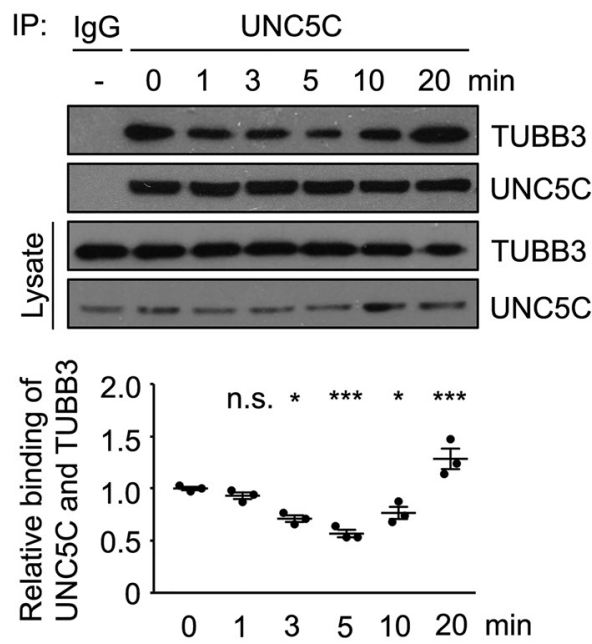

F

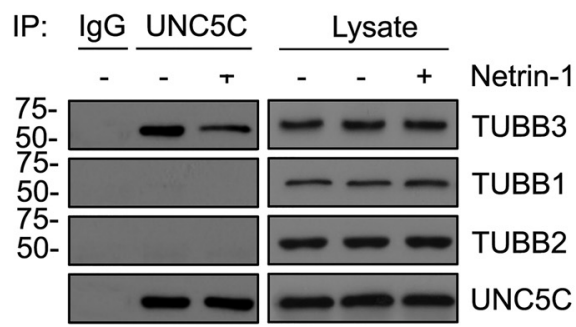

B

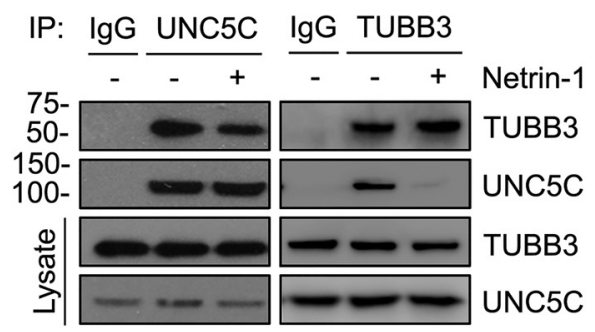

C

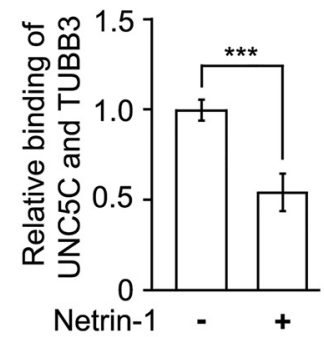

E
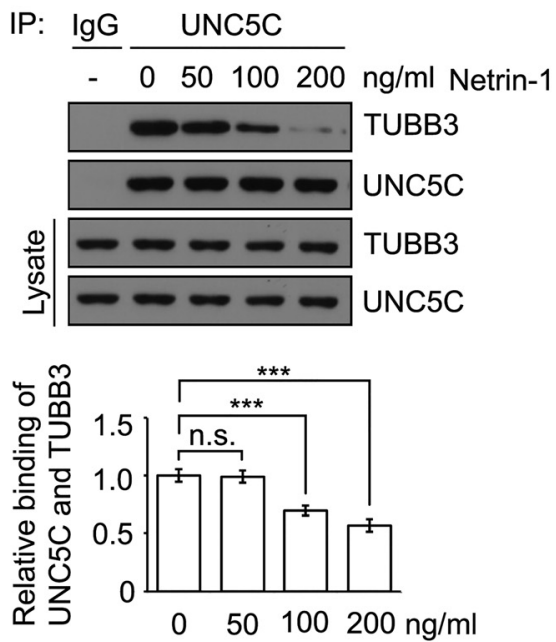

G

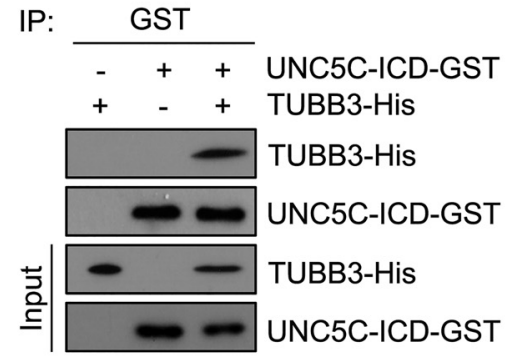

Figure 1. Netrin-1 reduces the interaction of UNC5C with TUBB3. A, UNC5C interacts with TUBB3 in HeLa cells. TUBB3-FLAG was cotransfected with UNC5C-HA into HeLa cells. Anti-HA (UNC5C) precipitated TUBB3-FLAG. B, Netrin-1 decreases the interaction of endogenous TUBB3 with UNC5C. Dissociated neurons from P4 mouse cerebella were stimulated with either the control or netrin-1-conditioned media (netrin-1 concentration was $\sim 300 \mathrm{ng} / \mu \mathrm{l}$ ) for $5 \mathrm{~min}$. Cell lysates were immunoprecipitated with either anti-UNC5C (left) or with anti-TUBB3 (right), and the immunoblot was analyzed with anti-TUBB3 and anti-UNC5C. C, Quantification of $\boldsymbol{B}$ from three independent experiments. ${ }^{* * *} p<0.001$ (two-tailed Student's $t$ test). $\boldsymbol{D}, \boldsymbol{E}$, Netrin-1 regulates the interaction of endogenous UNC5C with TUBB3 in a time-dependent $(\boldsymbol{D})$ and dose-dependent manner $(\boldsymbol{E})$. Primary neurons from P4 mouse cerebella were stimulated with either netrin-1-conditioned media (netrin-1 concentration was $\sim 300 \mathrm{ng} / \mu \mathrm{l}$ ) for $0-20 \mathrm{~min}(\boldsymbol{D})$ or purified netrin- 1 for 5 min $(\boldsymbol{E})$. Bottom, Quantification. ${ }^{*} p<0.05$ (one-way ANOVA and Tukey's test for post hoc comparisons). ${ }^{* * *} p<0.001$ (one-way ANOVA and Tukey's test for post hoc comparisons). $F$, Endogenous UNC5C interacts with TUBB3, not TUBB1 and TUBB2, in response of netrin-1 treatment. Primary neurons from P4 mouse cerebella were stimulated with either the control or netrin-1-conditioned media (netrin-1 concentration was $\sim 300 \mathrm{ng} / \mu \mathrm{l}$ ) for $5 \mathrm{~min}$. Cell lysates were immunoprecipitated with anti-UNC5C and followed by probing with anti-TUBB1, anti-TUBB2, or anti-TUBB3. G, Direct interaction of TUBB3 with UNC5C. Purified TUBB3 was incubated with purified intracellular domain of UNC5C tagged with GST in vitro. The anti-GST antibody was used to immunoprecipitate proteins, and the blot was analyzed with anti-TUBB3.

this result suggests that netrin-1 may reduce the interaction of UNC5C with dynamic polymerized TUBB3 in MTs. Taxol stabilizes MT against depolymerization in vitro. To further determine whether UNC5C interacts with polymeric TUBB3 in MTs, cell lysates from $\mathrm{P} 4$ mouse cerebellar neurons were treated with or without Taxol in vitro, and a MT cosedimentation assay was performed as previously described (Qu et al., 2013a; Huang et al.,
2015). In the absence of Taxol, MTs depolymerized in vitro and yielded monomerized TUBB3 subunits in the soluble supernatant and polymerized TUBB3 in the pellet (Fig. $3 C, D$ ). Addition of Taxol to cell lysates stabilized the polymerized MTs with more TUBB3 in the pellet than in the supernatant (Fig. 3C,D). Netrin-1 stimulation further increased pellet/supernatant fractionation of TUBB3 with or without Taxol (Fig. $3 C, D$ ), suggesting that 


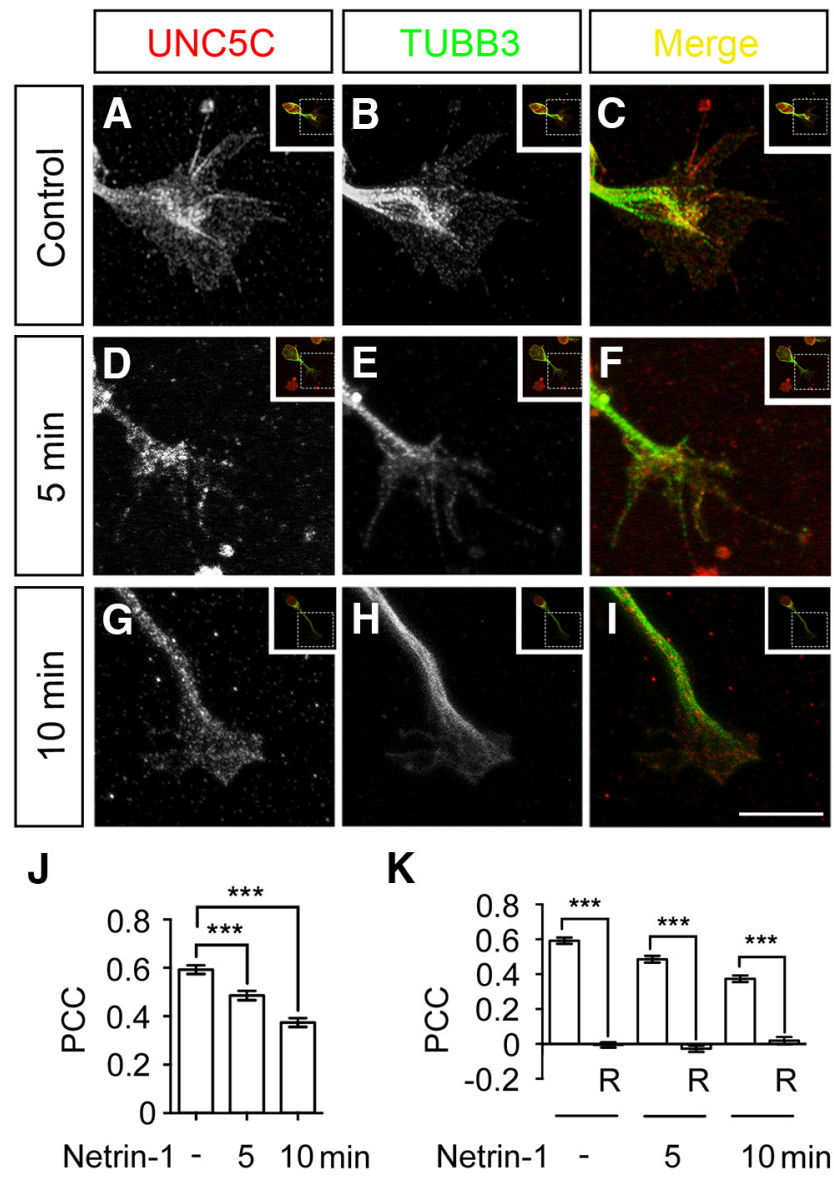

Figure 2. Netrin-1 reduces the subcellular overlap of UNC5C with TUBB3 in the GC of primary neurons. $\boldsymbol{A}-\boldsymbol{C}$, Overlap of immunofluorescent signals of UNC5C $(\boldsymbol{A})$ and TUBB3 $(\boldsymbol{B})$ in the GC of mouse EGL cells. P2 mouse EGL neurons were cultured for $20 \mathrm{~h}$ and stimulated with a shampurified control. C, Merged image of $\boldsymbol{A}, \boldsymbol{B}$. $\boldsymbol{A}-\boldsymbol{C}$, The value of $\mathrm{PCC}$ is $0.59 \pm 0.02$. D-I, 0verlap of endogenous UNC5C with TUBB3 in the GC of EGL neurons was reduced after netrin-1 stimulation for either $5 \min (\boldsymbol{D}-\boldsymbol{F})$ or $10 \mathrm{~min}(\boldsymbol{G}-\boldsymbol{I})$. $\boldsymbol{F}, \boldsymbol{I}$, Merged images of $\boldsymbol{D}, \boldsymbol{E}$ and $\boldsymbol{G}, \boldsymbol{H}$, respectively. $D-F$ and $\mathbf{G}-\boldsymbol{I}$, The value of PCC of UNC5C and TUBB3 is $0.49 \pm 0.02$ and $0.37 \pm 0.02$, respectively. Scale bar, $10 \mu \mathrm{m}$. J, Quantitative analysis of PCC in the control and netrin- 1 groups. A total of $37 \mathrm{GCs}$ in each group were analyzed. ${ }^{* * *} p<0.001$ (one-way ANOVA and Tukey's test for post hoc comparisons). $K$, Quantification of PCC after 90 degree counterclockwise rotation of one fluorescence channel. ${ }^{* *} p<0.001$ (one-way ANOVA and Tukey's test for post hoc comparisons). R, Rotation.

netrin-1 promotes MT polymerization in $\mathrm{P} 4$ cerebellar neurons. In the absence of Taxol, most of the endogenous UNC5C remained in the supernatant with or without netrin-1 (Fig. 3C,D). In contrast, Taxol treatment increased cosedimentation of UNC5C with MTs with more UNC5C in the pellet than in the supernatant (Fig. 3C,D). Netrin-1 reduced the amount of UNC5C in the MT-sedimented pellets (Fig. 3C,D). These results indicate that netrin-1 decreases the interaction of endogenous UNC5C with polymerized MTs. To further determine whether the netrin-1 effect depends on TUBB3, P4 cerebellar neurons were transfected with either TUBB3 control shRNA or TUBB3 shRNA and stimulated with netrin-1. A cosedimentation assay was performed in the presence of Taxol (Fig. 3E,F). As expected, netrin-1 stimulation yielded a significant amount of TUBB3 and UNC5C in the MT-sedimented pellets in the control shRNA group (Fig. $3 E, F$ ). Knockdown of TUBB3 abolished the effect of netrin-1 on cosedimentation of UNC5C with polymerized MTs (Fig. 3E,F). Together, these data indicate that netrin-1 reduces the interaction of endogenous UNC5C with polymerized TUBB3 in MTs.

\section{TUBB3 is required for netrin-1/UNC5C-mediated} axonal repulsion

To examine the role of TUBB3 in netrin-1 repulsion, primary EGL neurons from P2 mouse cerebella were transfected with Venus YFP with or without specific siRNA plasmids, and a Dunn chamber axon guidance assay was performed (Fig. 4A) (Yam et al., 2009). In this assay, the outer well was filled with either the sham-purified control or netrin-1, and transfected neurons immersed in the gradient right over the bridge area were examined. The angle turned was evaluated by measuring the angle between the initial and final position of an axon. As expected, in neurons transfected with Venus YFP alone or in combination with control shRNAs, netrin-1 repelled the axon (Fig. $4 B$, quantification in $C$ ). Our previous studies have shown that both UNC5C and TUBB3 shRNAs significantly reduce the level of endogenous UNC5C and TUBB3 in primary neurons, respectively (Fig. $3 E, G, H$ ) (Purohit et al., 2012; Qu et al., 2013a; Huang et al., 2015). Expression of either TUBB3 shRNA or UNC5C shRNA in primary P2 EGL neurons did not affect basal axon outgrowth (data not shown) but abolished netrin-1-induced axon repulsion (Fig. 4B, quantification in $C$ ). Importantly, expression of wild-type human TUBB3 or UNC5C rescued netrin-1-promoted axon repulsion in neurons treated with TUBB3 shRNA or UNC5C shRNA, respectively (Fig. $4 B$, quantification in $C$ ). These results indicate that TUBB3 is specifically involved in netrin-1/UNC5C-mediated repulsion.

\section{Netrin-1 differentially modulates MT dynamics in the GC} during axon repulsion

To examine MT dynamics in the GC during netrin-1-promoted repulsion, plasmids encoding GFP-tagged end-binding protein 3 (EB3-GFP), a marker of MT growth at the plus end in live cells, were transfected into P2 mouse cerebellar EGL cells, and a Dunn chamber axon guidance assay was performed in the presence of either a sham-purified control (Fig. $5 A, B$ ) or a netrin-1 gradient (Fig. $5 C, D$ ). MT growth in the GC was recorded by live-cell imaging of GFP-EB3, and kymographs were generated to track the movement of EB3 comets in the proximal (P) (close to the gradient) and the distal (D) (distal to the gradient) regions of the GC at 10,40, and $60 \mathrm{~min}$. The three time points represent the preturn, turning, and post-turn stages of the GC, respectively (Fig. $5 B, D$ ). The velocity (Fig. $5 E-G$, left panels) and travel distance (Fig. $5 E-G$, right panels) of the moving EB3-GFP comets were analyzed to assess MT dynamics in the $\mathrm{P}$ and $\mathrm{D}$ regions. As expected, the sham-purified control did not significantly change the velocity and travel distance of moving EB3 comets in the GC at all stages (Fig. 5A, B,E,F). Netrin-1, however, dramatically increased both the velocity and travel distance of EB3 comets in the $\mathrm{P}$ and $\mathrm{D}$ regions of the GC at the turning stage compared with the shampurified control group (Fig. $5 C, D, E-G$ ). Specifically, the velocity was increased from $0.23 \pm 0.01 \mu \mathrm{m} / \mathrm{s}$ and $0.21 \pm 0.02 \mu \mathrm{m} / \mathrm{s}$ without netrin- 1 to $0.29 \pm 0.01 \mu \mathrm{m} / \mathrm{s}$ and $0.33 \pm 0.02 \mu \mathrm{m} / \mathrm{s}$ with netrin- 1 in the $\mathrm{P}$ and $\mathrm{D}$ regions, respectively; the distance was increased from $1.02 \pm 0.04 \mu \mathrm{m}$ and $1.15 \pm 0.06 \mu \mathrm{m}$ without netrin- 1 to $1.30 \pm 0.06 \mu \mathrm{m}$ and $1.39 \pm 0.03 \mu \mathrm{m}$ with netrin- 1 in the $\mathrm{P}$ and in D regions, respectively. Netrin-1 did not significantly alter the velocity and travel distance of EB3 comets in both $\mathrm{P}$ and $D$ regions at the preturn or post-turn stage (Fig. $5 E-G$ ). Interestingly, the velocity and travel distance of moving EB3 comets in the $\mathrm{P}$ and $\mathrm{D}$ regions were differentially regulated by netrin-1 during GC turning: with a netrin-1 gradient, the velocity and travel distance of moving EB3 comets were increased from $0.29 \pm 0.01$ $\mu \mathrm{m} / \mathrm{s}$ to $0.33 \pm 0.02 \mu \mathrm{m} / \mathrm{s}$ and from $1.30 \pm 0.06 \mu \mathrm{m}$ to $1.39 \pm$ 
A

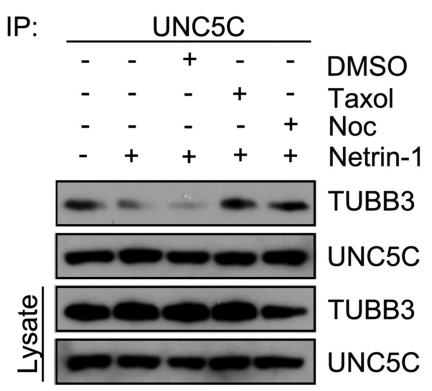

C

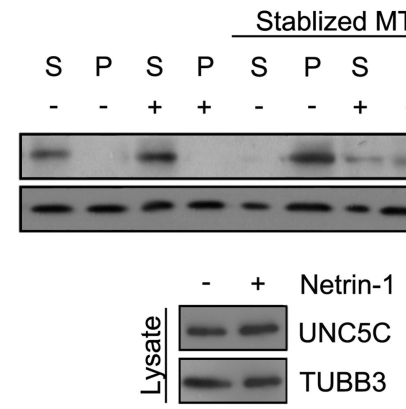

$\mathbf{E}$
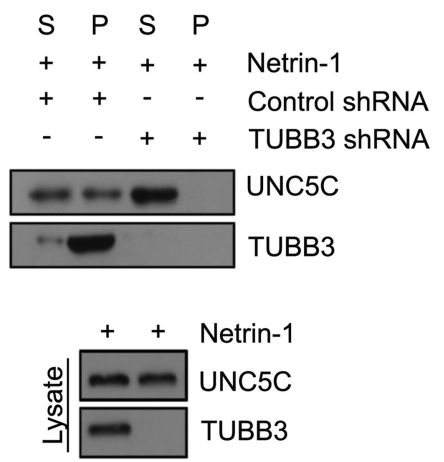

B

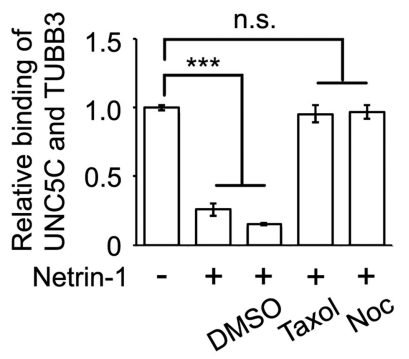

D

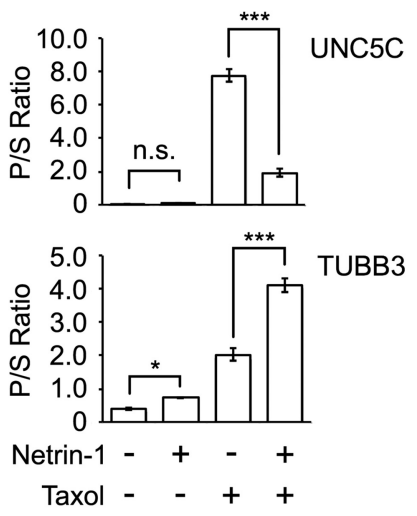

$\mathbf{F}$

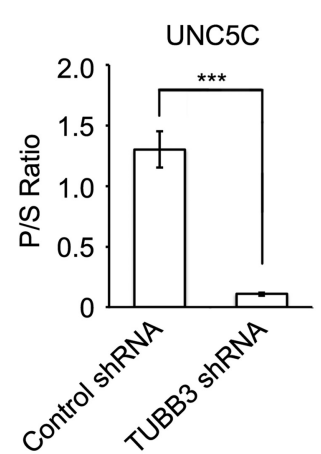

G

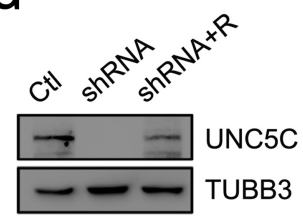

H

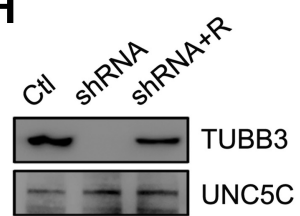

Figure 3. Netrin-1 decreases the interaction of UNC5C with polymerized TUBB3. A, Taxol and nocodazole (Noc) inhibited the netrin-1-induced UNC5C/TUBB3 dissociation. Dissociated P4 mouse cerebellar neurons were treated with purified netrin-1 in the presence of $1 \mu \mathrm{m}$ Taxol, $3 \mu \mathrm{m}$ nocodazole, or DMSO. $\boldsymbol{B}$, Quantification of $\boldsymbol{A}$ from three independent experiments showing relative binding of UNC5C to TUBB3. ${ }^{* * *} p<0.001$ (one-way ANOVA and Tukey's test for post hoc comparisons). C, Primary P4 mouse cerebellar neurons were stimulated with netrin-1 or sham-purified control, and a cosedimentation assay of cell lysates was performed in the absence or presence of Taxol. UNC5C and TUBB3 in the pellet $(P)$ and supernatant $(S)$ fractions were examined by immunoblotting using anti-UNC5C and anti-TUBB3 antibodies, respectively. D, Quantification of $\mathbf{C}$ from three independent experiments showing P/S ratio of UNC5C and TUBB3. ${ }^{*} p<0.05$ (one-way ANOVA and Tukey's test for post hoc comparisons). ${ }^{* * *} p<$ 0.001 (one-way ANOVA and Tukey's test for post hoc comparisons). $\boldsymbol{E}$, Dissociated P4 mouse cerebellar neurons were transfected with either TUBB3 shRNA or control shRNA and stimulated with purified netrin-1. The cosedimentation assay was conducted with Taxol to stabilize MTs in vitro as above. $\boldsymbol{F}$, Quantification of $P / S$ ratio of $\boldsymbol{E}$ from three independent experiments. ${ }^{* *} p<0.001$ (two-tailed Student's t test). G, Knockdown of endogenous UNC5C in primary neurons. P4 mouse cerebellar neurons were nucleofected with control shRNA, UNC5C shRNA, or UNC5C shRNA plus wild-type human UNC5C. Ctl, Control shRNA; R, wild-type human UNC5C. $\boldsymbol{H}$, TUBB3 shRNA specifically knocked down endogenous TUBB3. P4 mouse cerebellar neurons were transfected with control shRNA, TUBB3 shRNA, or TUBB3 plus wild-type human TUBB3. Ctl, Control shRNA; R, wild-type human TUBB3.

$0.03 \mu \mathrm{m}$ in the $\mathrm{P}$ and in $\mathrm{D}$ regions, respectively. In the presence of a netrin-1 gradient, the $\mathrm{P} / \mathrm{D}$ ratios of comet velocity and travel distance at the preturn and post-turn stage were quite stable at $\sim 1.0$. However, netrin-1 dramatically reduced both $\mathrm{P} / \mathrm{D}$ ratios at the turning stage: the $\mathrm{P} / \mathrm{D}$ ratios of velocity and travel distance were $0.77 \pm 0.09$ and $0.83 \pm 0.05$ at 40 min, respectively (Fig. $5 E-G$ ). These data indicate that netrin-1 differentially increases MT dynamics in the GC during axon repulsion with more $\mathrm{MT}$ growth in the $\mathrm{D}$ than the $\mathrm{P}$ region.

TUBB3 is specifically involved in netrin-1/UNC5C-regulated MT dynamics in the GC

To examine whether UNC5C is required for netrin-1-mediated MT dynamics in the GC, EB3-GFP was cotransfected with control shRNA (Fig. 6A), UNC5C shRNA (Fig. $6 B$ ), or UNC5C shRNA plus wildtype human UNC5C (Fig. 6C) into primary EGL neurons from P2 mouse cerebella. Next, MT dynamics in the GC were recorded by live-cell imaging of GFP-EB3 in the presence of a netrin-1 gradient. In EGL cells transfected with the control shRNA, netrin-1 differentially regulated MT growth in the turning GC with more MT growth in the D than the $\mathrm{P}$ region (Fig. 6A, quantification in $F$ ). Knockdown of endogenous UNC5C not only inhibited netrin-1-increased MT growth in the GC but abolished the differential induction of MT dynamics in the D region, compared with the control shRNA group (Fig. $6 B$, quantification in $F$ ): the velocity of moving GFP-EB3 comets in the $\mathrm{P}$ and $\mathrm{D}$ regions was decreased from $0.29 \pm 0.01 \mu \mathrm{m} / \mathrm{s}$ and $0.32 \pm 0.02 \mu \mathrm{m} / \mathrm{s}$ in the control shRNA group to $0.26 \pm 0.01$ $\mu \mathrm{m} / \mathrm{s}$ and $0.27 \pm 0.01 \mu \mathrm{m} / \mathrm{s}$ in the UNC5C shRNA group, respectively; the travel distance of EB3 comets in the P and in D regions was decreased from $1.31 \pm 0.03$ $\mu \mathrm{m}$ and $1.41 \pm 0.03 \mu \mathrm{m}$ in the control shRNA group to $0.97 \pm 0.04 \mu \mathrm{m}$ and $0.99 \pm 0.04 \mu \mathrm{m}$ in the UNC5C shRNA group, respectively. Expression of wildtype human UNC5C rescued the effect of UNC5C knockdown on netrin-1-induced MT dynamics (Fig. 6C, quantification in $F)$. These results indicate that UNC5C is specifically involved in netrin-1-induced MT dynamics in the GC of primary EGL neurons. To further determine whether TUBB3 is required for netrin-1-regulated MT dynamics during axon repulsion, EB3-GFP was cotransfected with control shRNA, TUBB3 shRNA, or TUBB3 shRNA together with wild-type human TUBB3 into mouse P2 cerebellar EGL cells and the Dunn chamber axon guidance assay performed to track the movement of GFP-EB3 comets in the GC in the presence of a netrin-1 gradient. In neurons transfected with control shRNA, netrin-1 differentially induced MT growth in the GC (Fig. 6A, quantification in $F$ ). In contrast, expression of TUBB3 shRNA abolished netrin-1-induced MT growth in both D and P regions of the GC 
A

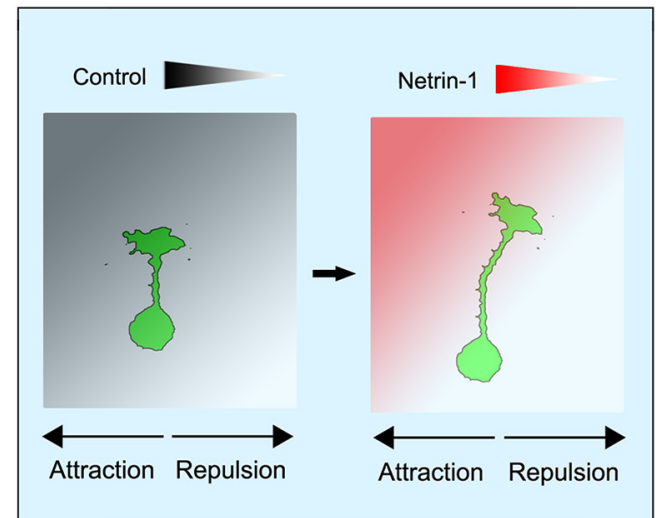

C

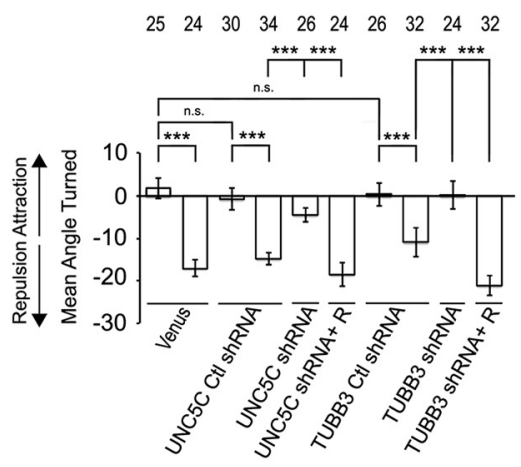

Netrin Gradient - + - + + + - + + +
B
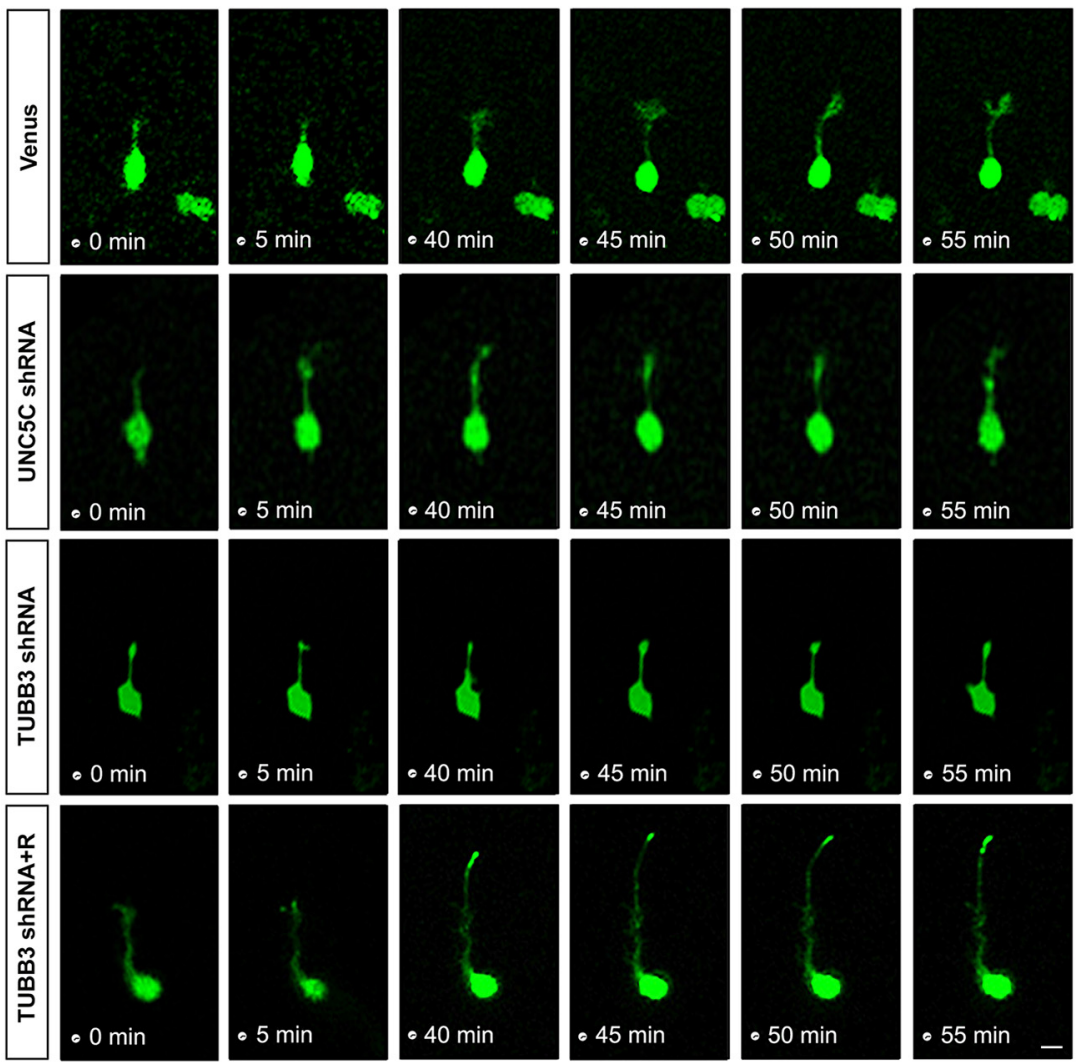

Figure 4. TUBB3 is required for netrin-1/UNC5C-mediated axon repulsion. $A$, Schematic diagram of axon turning of P2 mouse EGL cells in a control (left) or netrin-1 (right) gradient using a Dunn chamber axon guidance assay. $\boldsymbol{B}$, Live-cell imaging showing axon turning of P2 EGL cells transfected with Venus-YFP only or combination of Venus-YFP with other indicated constructs. Nucleofection of Venus-YFP into P2 EGL neurons allowed visualization of axon projection. A left-to-right netrin-1 gradient was established as shown in $\boldsymbol{A}$. Scale bar, $10 \mu \mathrm{m}$. $\boldsymbol{C}$, Quantification of axon turning of P2 EGL neurons. Data are mean \pm SEM. ${ }^{* * *} p<0.001$ (one-way ANOVA and Tukey's test for post hoc comparisons). The numbers on the top of each bar indicate the numbers of GCs analyzed in the corresponding groups.

(Fig. 6D, quantification in $F$ ): the velocity of moving GFP-EB3 comets in the $\mathrm{P}$ and $\mathrm{D}$ region was decreased from $0.29 \pm 0.01$ $\mu \mathrm{m} / \mathrm{s}$ and $0.32 \pm 0.02 \mu \mathrm{m} / \mathrm{s}$ in the control shRNA group to $0.26 \pm 0.01 \mu \mathrm{m} / \mathrm{s}$ and $0.27 \pm 0.02 \mu \mathrm{m} / \mathrm{s}$ in the TUBB3 shRNA group, respectively; the travel distance of EB3 comets in the P and in $\mathrm{D}$ region was decreased from $1.31 \pm 0.03 \mu \mathrm{m}$ and $1.41 \pm 0.03$ $\mu \mathrm{m}$ in the control shRNA group to $1.07 \pm 0.07 \mu \mathrm{m}$ and $1.14 \pm$ $0.04 \mu \mathrm{m}$ in the TUBB3 shRNA group, respectively. The effect of TUBB3 shRNA on netrin-1-regulated MT dynamics in the GC was rescued by cotransfecting the wild-type human TUBB3 plasmids (Fig. $6 E$, quantification in $F$ ): the velocity of moving GFPEB3 comets was $0.29 \pm 0.01 \mu \mathrm{m} / \mathrm{s}$ in the $\mathrm{P}$ region and $0.33 \pm 0.01$ $\mu \mathrm{m} / \mathrm{s}$ in the $\mathrm{D}$ region; the travel distance of EB3 comets was $1.31 \pm 0.02 \mu \mathrm{m}$ in the $\mathrm{P}$ region and $1.44 \pm 0.07 \mu \mathrm{m}$ in the $\mathrm{D}$ region. Together, these results support the hypothesis that TUBB3 is specifically involved in netrin-1/UNC5C-induced MT dynamics in the GC during axon repulsion.

\section{TUBB3 is required for axon projection of DRG neurons in vivo}

A ventral-to-dorsal gradient of netrin-1 derived from the floor plate repels DRG axon projection, prevents the DRG axons from entering aberrantly into the intermediate and ventral regions of the spinal cord, and guides them to project precisely toward the DREZ (Kennedy et al., 2006; Masuda et al., 2008, 2009). Transient expression of netrin-1 in the dorsal spinal cord, acting as a short-range inhibitory cue, may elaborate the "waiting period" for extension of sensory afferents and prevent premature DRG axons from entering into the mantle layer of the spinal cord (Watanabe et al., 2006). To further reveal the role of netrin-1 in DRG axon pathfinding in vivo, Venus YFP was introduced either alone or with AP-netrin-1 into the chick neural tube at Stage 16 (Fig. $7 B, C^{\prime \prime}$ ) as previously described (Masuda et al., 2008). YFPlabeled embryos at Stages 23-25 were collected, and transverse sections of the lumbosacral segments of the spinal cords were immunostained with axonal-specific BEN antibodies (SC1/DMGRASP protein) to label DRG axons (Avraham et al., 2010). In embryos expressing Venus YFP only (Fig. $7 B-B^{\prime \prime}$ ), DRG axons projected normally toward and eventually entered the dorsal spinal cord at the DREZ (Fig. $7 A-B^{\prime \prime}$, quantification in $J$ ). Overexpression of netrin- 1 in the dorsal and intermediate regions of the spinal cord (Fig. $7 C-C^{\prime \prime}$ ) increased DREZ size (quantification in Fig. $7 J$ ), suggesting that DRG axons may enter aberrantly into the spinal cord. Three possible mechanisms could cause this guidance defect: (1) ectopic expression of netrin-1 in the dorsal and intermediate regions of the spinal cord disrupts the ventral-todorsal gradient of netrin-1, allowing axons enter into the spinal cord more ventrally; (2) overexpression of netrin-1 in the dorsal spinal cord could extend the "waiting period" of DRG axon projection into mantle layer of the spinal cord, increasing DREZ size; and (3) overexpression of netrin-1 in the spinal cord could desensitize DRG axons to netrin-1 (adaptation), resulting in the axon projection defect. To determine whether UNC5C is required for DRG axon projection in vivo, Venus YFP was electroporated either alone or with UNC5C control shRNA, UNC5C shRNA, and UNC5C shRNA plus wild-type human UNC5C into 
A
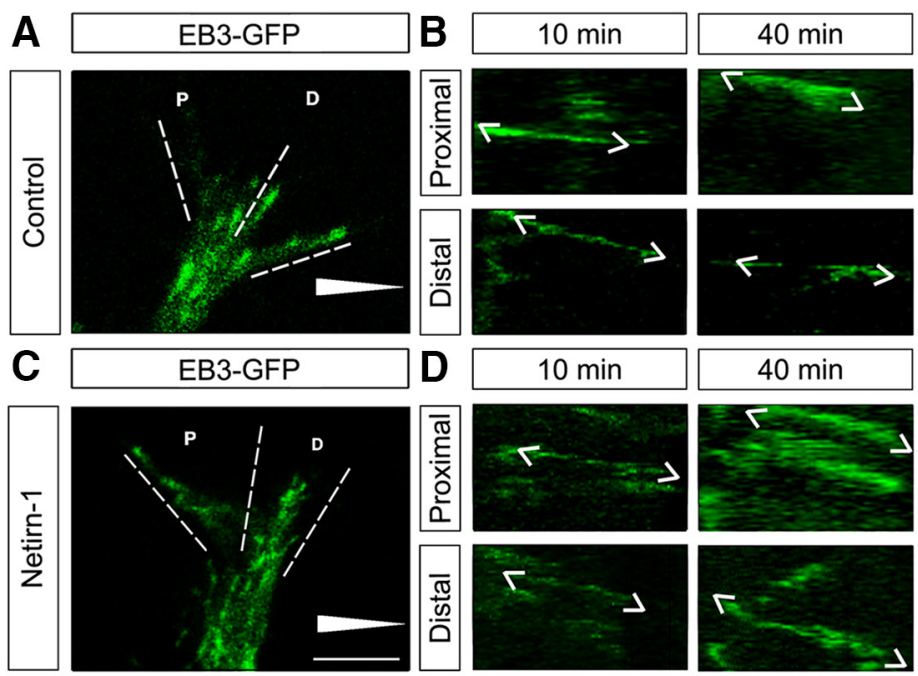

$E$
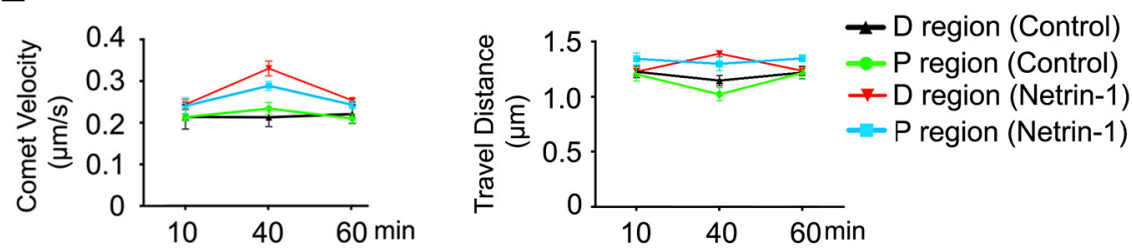

$\mathbf{F}$
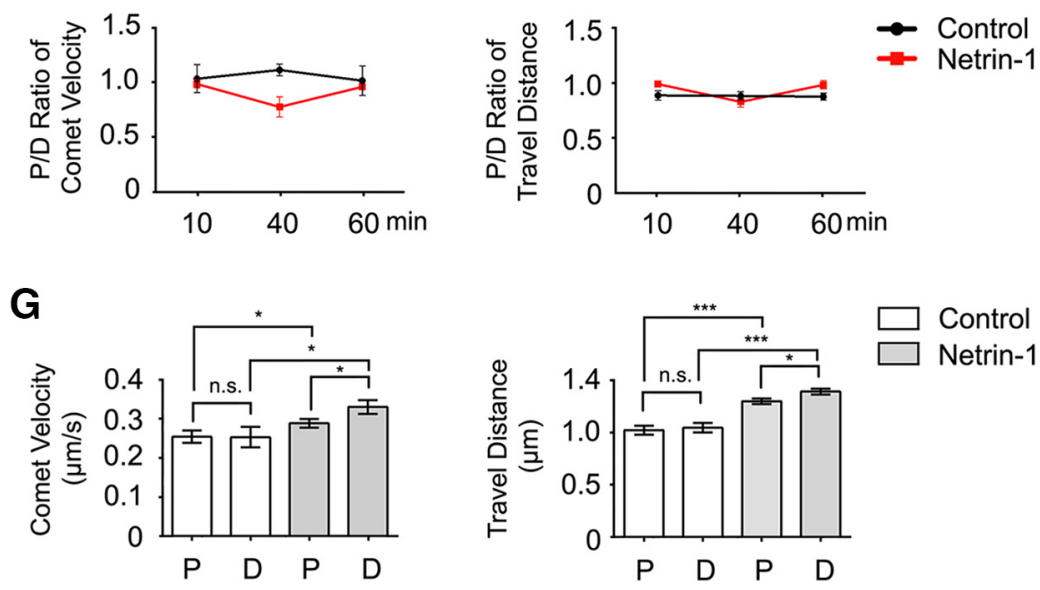

Figure 5. Visualization of MT dynamics in the GC via time-lapse recording of EB3-GFP in primary neurons during axon turning $A-D$, Trajectories of EB3-GFP comets in the GC of P2 mouse EGL neurons. P2 mouse EGL neurons were dissociated and nucleofected with EB3-GFP. Primary neurons were cultured for $2 \mathrm{~d}$, and a Dunn chamber axon guidance assay was performed in the absence $(\boldsymbol{A}, \boldsymbol{B})$ or presence of a netrin-1 gradient $(\boldsymbol{C}, \boldsymbol{D}) . \boldsymbol{B}, \boldsymbol{D}$, Kymographs of EB3-GFP comet movements in the proximal (upper) and distal regions (lower) of the $\mathrm{GCs} \sin A$ and $C$, respectively. Scale bar, $10 \mu \mathrm{m}$. White triangle represents either a control or netrin- 1 gradient. $\boldsymbol{E}, \boldsymbol{F}$, Quantification of velocity ( $\boldsymbol{E}$, left) and travel distance $(\boldsymbol{E}$, right) as well as ratios of velocity ( $\boldsymbol{F}$, left) and travel distance $(\boldsymbol{F}$, right) of moving EB3-GFP comets in proximal and distal regions of the GC of P2 EGL cells $(\boldsymbol{A}-\boldsymbol{D}) . \mathbf{G}$, Bar graph comparisons of the velocity (left) and travel distance (right) of moving EB3-GFP comets in the GC of P2 EGL cells at 40 min after image capture from $\boldsymbol{A}-\boldsymbol{D}$. Data are mean \pm SEM ( $3 \mathrm{GCS}$ in each group). ${ }^{*} p<0.05$ (one-way ANOVA with Tukey's test for post hoc comparisons). ${ }^{* * *} p<0.001$ (one-way ANOVA with Tukey's test for post hoc comparisons).

the chick neural tube as Stage 12-15, a time when the neural crest cells emigrate from the chick spinal cord (Bronner-Fraser and Fraser, 1989; Serbedzija et al., 1989; Ruffins et al., 1998), and transverse sections of the lumbosacral segments of the spinal cords after electroporation were prepared and immunostained with BEN antibodies. UNC5C shRNA (Fig. 7E- $E^{\prime \prime}$ ), but not UNC5C control shRNA (Fig. 7D-D ${ }^{\prime \prime}$ ), significantly increased DREZ size (quantification in Fig. $7 J$ ). Expression of wild-type

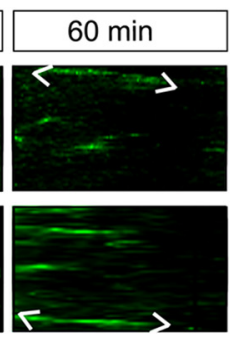

$60 \mathrm{~min}$
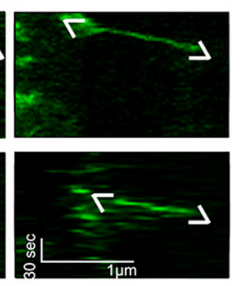

human UNC5C rescued the effect of UNC5C knockdown on DRG axon projection toward the spinal cord (Fig. $7 F-F^{\prime \prime}$, quantification in $J$ ). These results indicate that netrin-1/UNC5C signaling is required for proper DRG axon pathfinding in vivo. To reveal the role of TUBB3 in DRG axon projection, we introduced $\mathrm{Ve}$ nus YFP with TUBB3 control shRNA, Venus YFP with TUBB3 shRNA, or Venus YFP with TUBB3 shRNA plus wild-type human TUBB3 together into the chick spinal cord by in ovo electroporation. TUBB3 control shRNA did not affect DRG axon projection toward the spinal cord with normal DREZ size compared with the Venus YFP group (Fig. $7 G-G^{\prime \prime}$, quantification in $J$ ). However, knockdown of TUBB3 dramatically increased DREZ size (Fig. $7 H-H^{\prime \prime}$, quantification in $J$ ). The phenotype of TUBB3 knockdown on DRG axon projection was reversed by cotransfecting wild-type human TUBB3 plasmids (Fig. $7 I-I^{\prime \prime}$, quantification in $J$ ). These results demonstrate that TUBB3 is required for the DRG axon projection and pathfinding in the developing spinal cord.

\section{Discussion}

In the developing GC, MT dynamic instability, a spontaneous switch between phases of growth and shortening, may function as a direct sensor to control GC steering (Tanaka and Sabry, 1995; Suter and Forscher, 2000; Buck and Zheng, 2002; Dent et al., 2004; Conde and Cáceres, 2009; Dent et al., 2011; Liu and Dwyer, 2014). Our recent studies demonstrate that direct coupling of DCC and DSCAM to MT dynamics via TUBB3 is required for netrin-1-promoted axon outgrowth, branching, and attraction $(\mathrm{Qu}$ et al., 2013a; Huang et al., 2015). The results here suggest that uncoupling of UNC5C with polymerized MTs via TUBB3 plays an important role in netrin-1-mediated repulsion.

\section{Disengagement of UNC5C with polymerized TUBB3 in netrin- 1 repulsion}

In this study, we have found that UNC5C interacts directly with TUBB3, and netrin-1 decreases this interaction both in vitro and in vivo (Fig. 1). TUBB3 colocalizes with UNC5C in the GC of developing cerebellar EGL cells, and netrin-1 further reduces this colocalization (Fig. 2). Disruption of MT dynamics with either Taxol or nocodazole abolishes the netrin-1-reduced interaction of endogenous UNC5C with TUBB3, suggesting that MT dynamics are required for the netrin-1-regulated interaction (Fig. $3 A, B$ ). UNC5C cosediments with polymerized MTs from primary cerebellar neuron lysates, and netrin-1 reduces this cosedimentation 
A

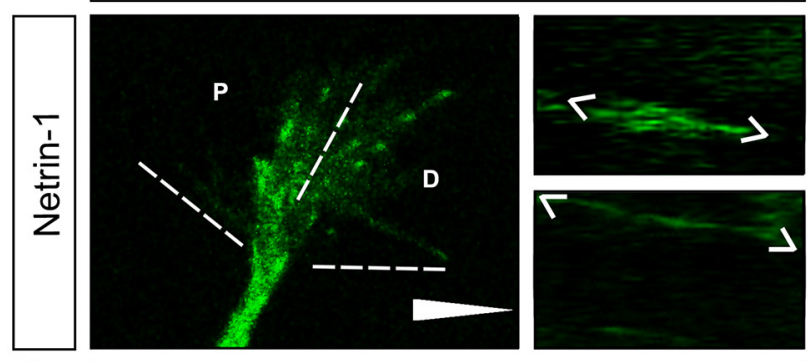

D
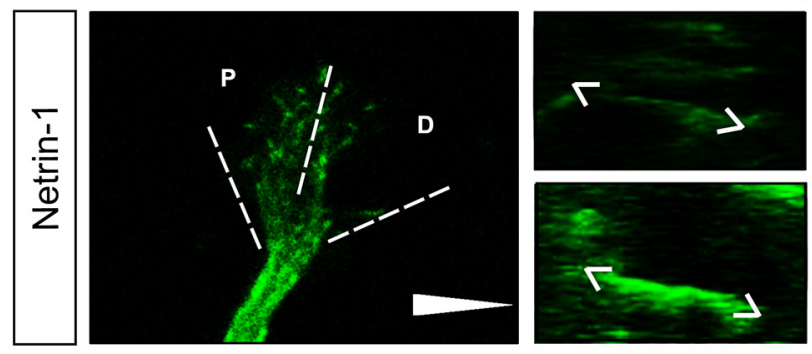

B

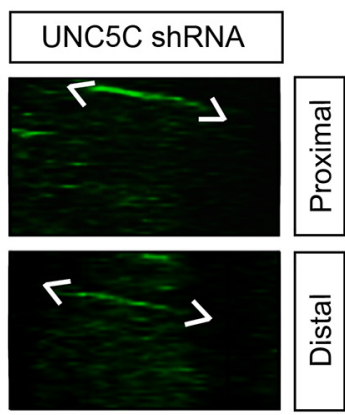

C UNC5C shRNA+R

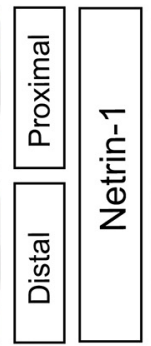

E
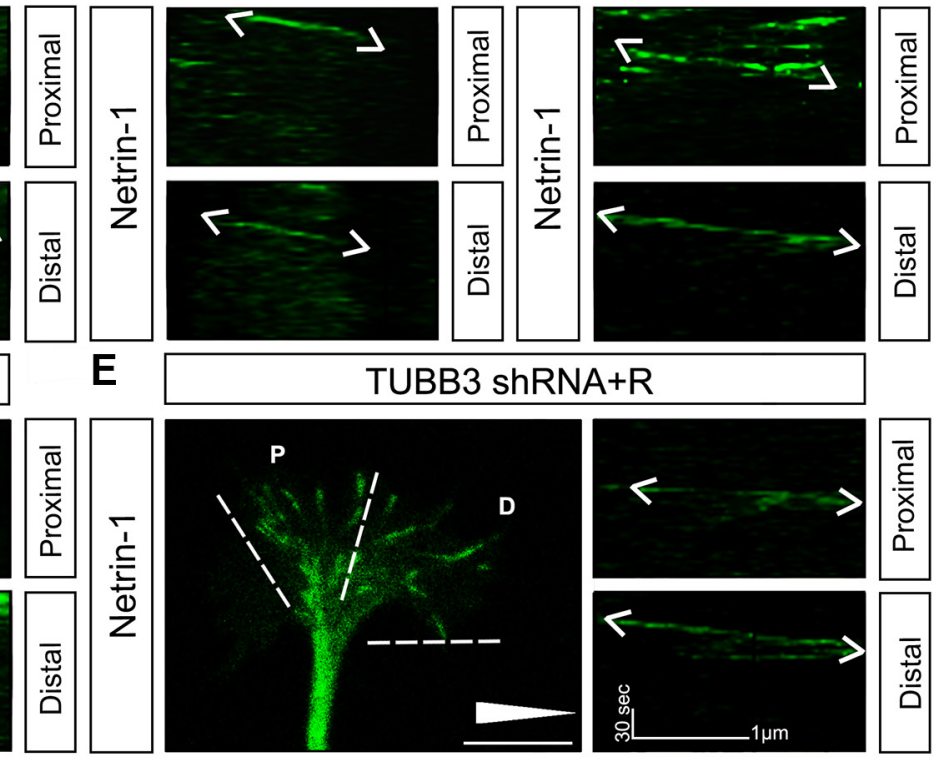

$A+R$

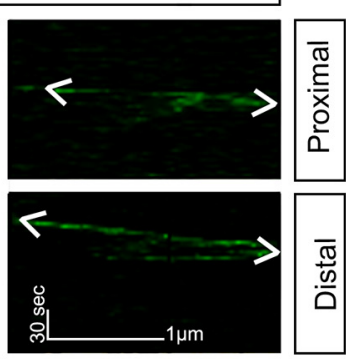

F
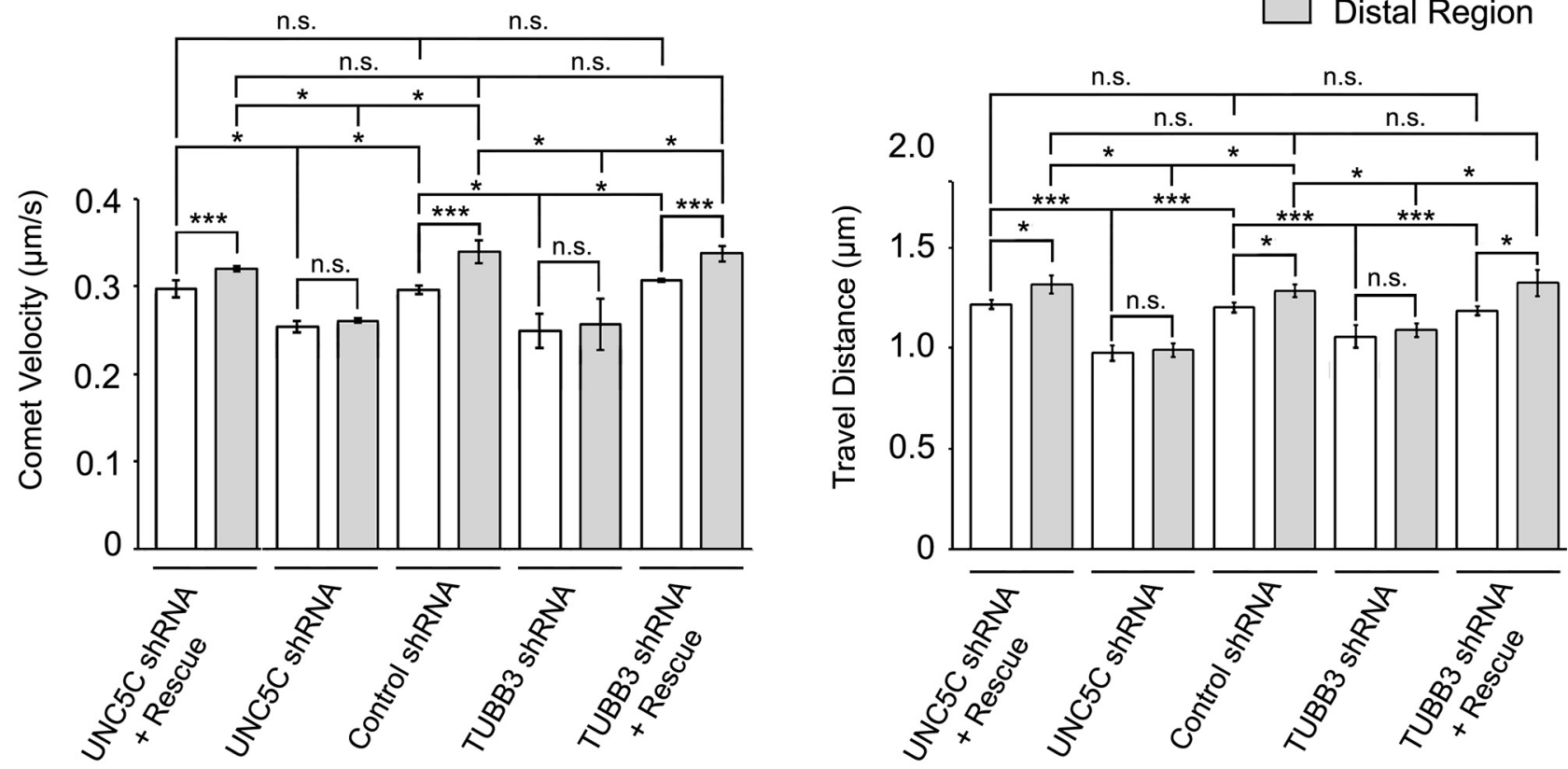

Figure 6. TUBB3 is specifically involved in netrin-1/UNC5C-regulated MT dynamics in the GC of P2 cerebellar EGL neurons. A-E, P2 mouse EGL neurons were cotransfected EB3-GFP with control shRNA ( $\boldsymbol{A})$, UNC5 ( shRNA (B), UNC5 ( shRNA plus wild-type human UNC5C ( $($ ), TUBB3 shRNA (D), or TUBB3 shRNA plus wild-type human TUBB3 (E), respectively, and a Dunn chamber axon guidance assay was performed. $A, D, E$, Left, Live-cell imaging tracking EB3-GFP comet movements in the GC. Right, Kymographs of EB3-GFP comets in the proximal (upper) and distal (lower) regions of the GC. $\boldsymbol{B}, \boldsymbol{C}$, Kymographs of EB3-GFP comet movements in the proximal (upper) and distal (lower) regions of the GC of P2 cerebellar EGL cells transfected with UNC5C shRNA ( $\boldsymbol{B}$ ) or UNC5C shRNA plus wild-type human UNC5C (C). Scale bar, $10 \mu \mathrm{m}$. White triangle represents either a control or netrin-1 gradient. $\boldsymbol{F}$, Quantification of the velocity (left) and travel distance (right) of moving EB3-GFP comets in the GC of P2 EGL cells $(\boldsymbol{A}-\boldsymbol{E})$ at 40 min after image capture. Data are mean \pm SEM (3 GCs in each group). ${ }^{*} p<0.05$ (one-way ANOVA with Tukey's test for post hoc comparisons). ${ }^{* * *} p<0.001$ (one-way ANOVA with Tukey's test for post hoc comparisons).

with less UNC5C in the pellet than the supernatant (Fig. 3C,D). Netrin-1-reduced cosedimentation of UNC5C with polymerized MTs is dependent on TUBB3 (Fig. 3C-F). Intriguingly, netrin-1 stimulation increases the ratio of polymerized TUBB3 in the pellet (Fig. $3 C, D$ ). These results suggest that netrin-1 may promote MT dynamics through the UNC5C/TUBB3 interaction. Our functional data indicate that TUBB3 is specifically involved in netrin-1- mediated axon repulsion both in vitro and in vivo (Figs. 4, 7). More importantly, data from live-cell imaging indicate that netrin-1 differentially increases MT dynamics in the GC of cerebellar EGL neurons with more MT growth in the distal region than the proximal region, and knockdown of either UNC5C or TUBB3 abolishes the netrin-1-promoted differential MT growth in the GC during axon turning (Figs. 5, 6). These results indicate 

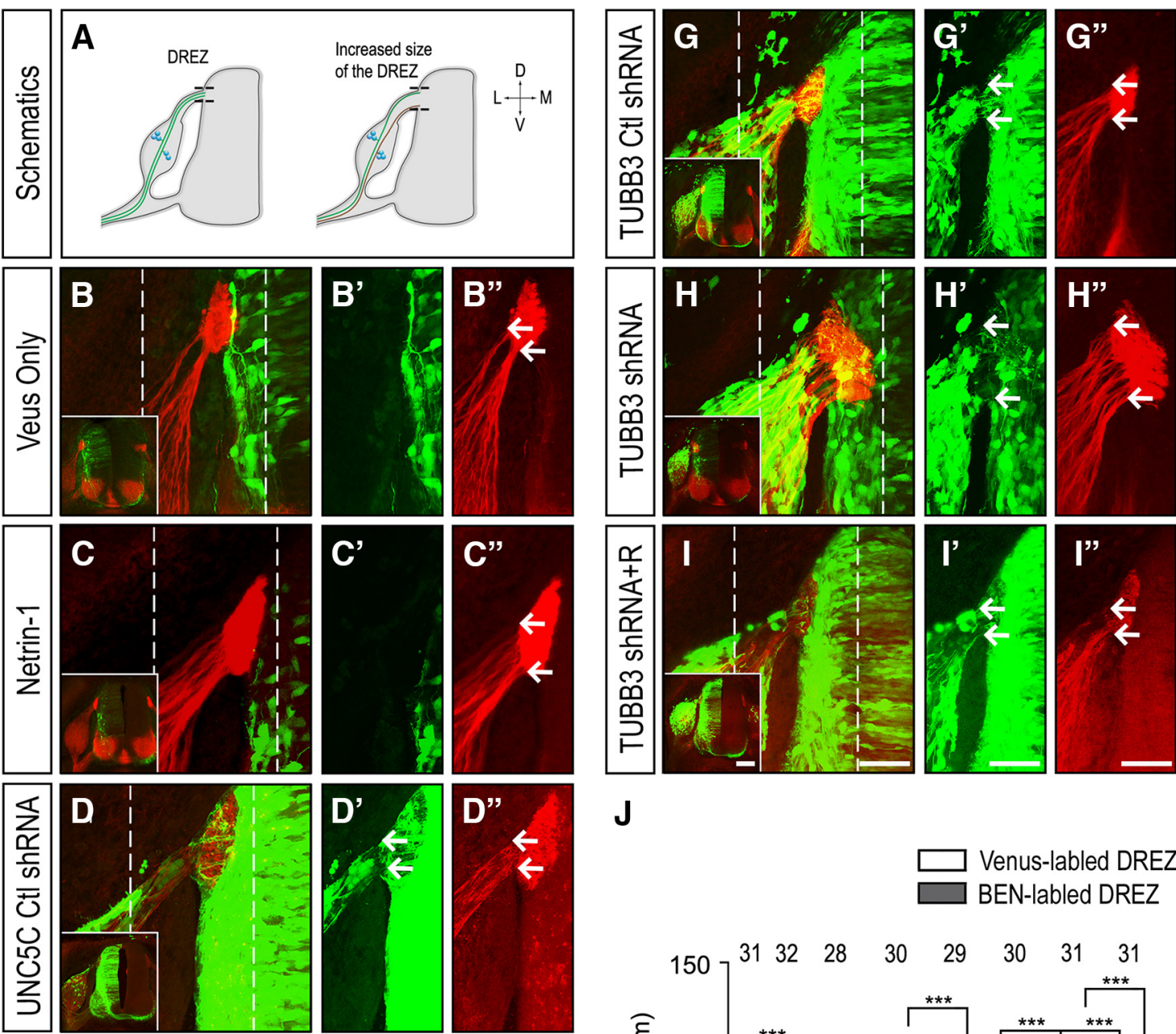

$\mathbf{J}$
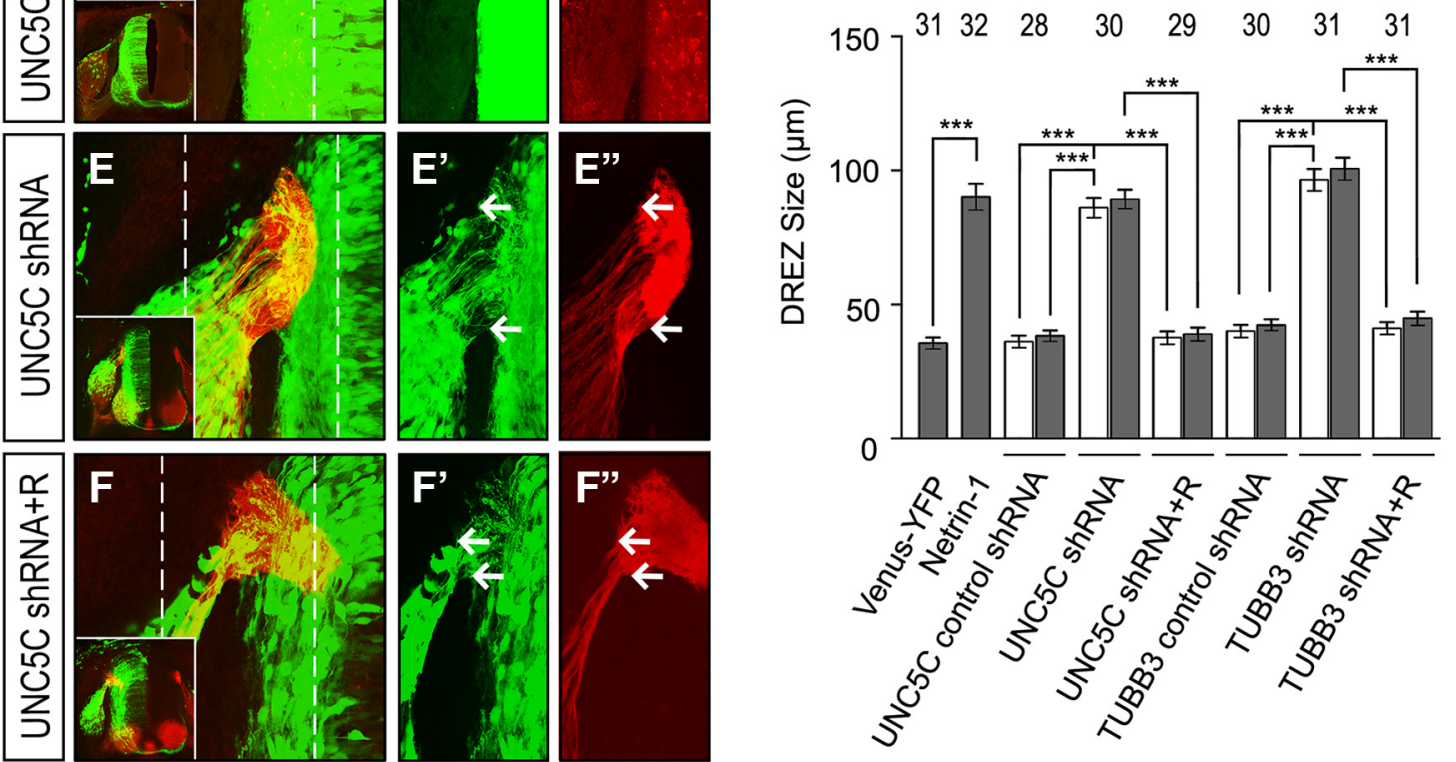

Figure 7. TUBB3 is essential for DRG axon projection in vivo. $A$, Schematic showing DRG axon projection toward the chick spinal cord after electroporation. Brown represents DRG axon bundles with projection deficiency. Green represents normal DRG axon bundles. $\boldsymbol{B}-\boldsymbol{C}^{\prime \prime}$, The chickneural tube at Stage 16 was electroporated with Venus YFP only $\left(\boldsymbol{B}-\boldsymbol{B}^{\prime \prime}\right)$ or Venus YFP plus AP-netrin-1 (C- $\left.\boldsymbol{C}^{\prime \prime}\right)$, and transverse sections of the spinal cord were stained with BEN antibody (red). $\boldsymbol{D}-\boldsymbol{I}^{\prime \prime}$, The chick neural tube at Stages $12-15$ was electroporated with Venus YFP plus UNC5C control shRNA (D-D $\mathbf{D}^{\prime \prime}$ ), Venus YFP plus UNC5( shRNA $\left(\boldsymbol{E}-\boldsymbol{E}^{\prime \prime}\right)$, Venus YFP plus UNC5( shRNA and wild-type human UNC5C $\left(\boldsymbol{F}-\boldsymbol{F}^{\prime \prime}\right)$, Venus YFP plus TUBB3 control shRNA ( $\left.\boldsymbol{G}-\boldsymbol{G}^{\prime \prime}\right)$, Venus YFP plus TUBB3 shRNA $\left(\boldsymbol{H}-\boldsymbol{H}^{\prime \prime}\right)$, Venus YFP plus TUBB3 shRNA and wild-type human TUBB3 $\left(\boldsymbol{I}-\boldsymbol{I}^{\prime \prime}\right)$. Transverse sections of the Venus YFP-labeled spinal cords were stained with BEN antibody. B- $\boldsymbol{I}$, Overlay confocal images of green (Venus YFP) and red (BEN staining) fluorescence. Insets, Corresponding low-magnification images of $\boldsymbol{B}-\boldsymbol{I}$. $\boldsymbol{B}^{\prime}-\boldsymbol{I}^{\prime}, \boldsymbol{B}^{\prime \prime}-\boldsymbol{I}^{\prime \prime}$, Venus YFP images and images of BEN antibody immunostaining of the region of interest in $\boldsymbol{B}-\boldsymbol{I}$ (dashed lines), respectively. Scale bar, $50 \mu \mathrm{m}$. J, Quantification of DREZ size. ${ }^{* * *} p<0.001$ (one-way ANOVA with Tukey's test for post hoc comparisons). Both Venus YFP and BEN antibody staining consistently demarcates DRG axon projections with no statistically significant difference in DREZ size in each group. The numbers on the top of each bar indicate the numbers of samples tested in the corresponding groups. R, Rescue constructs. 


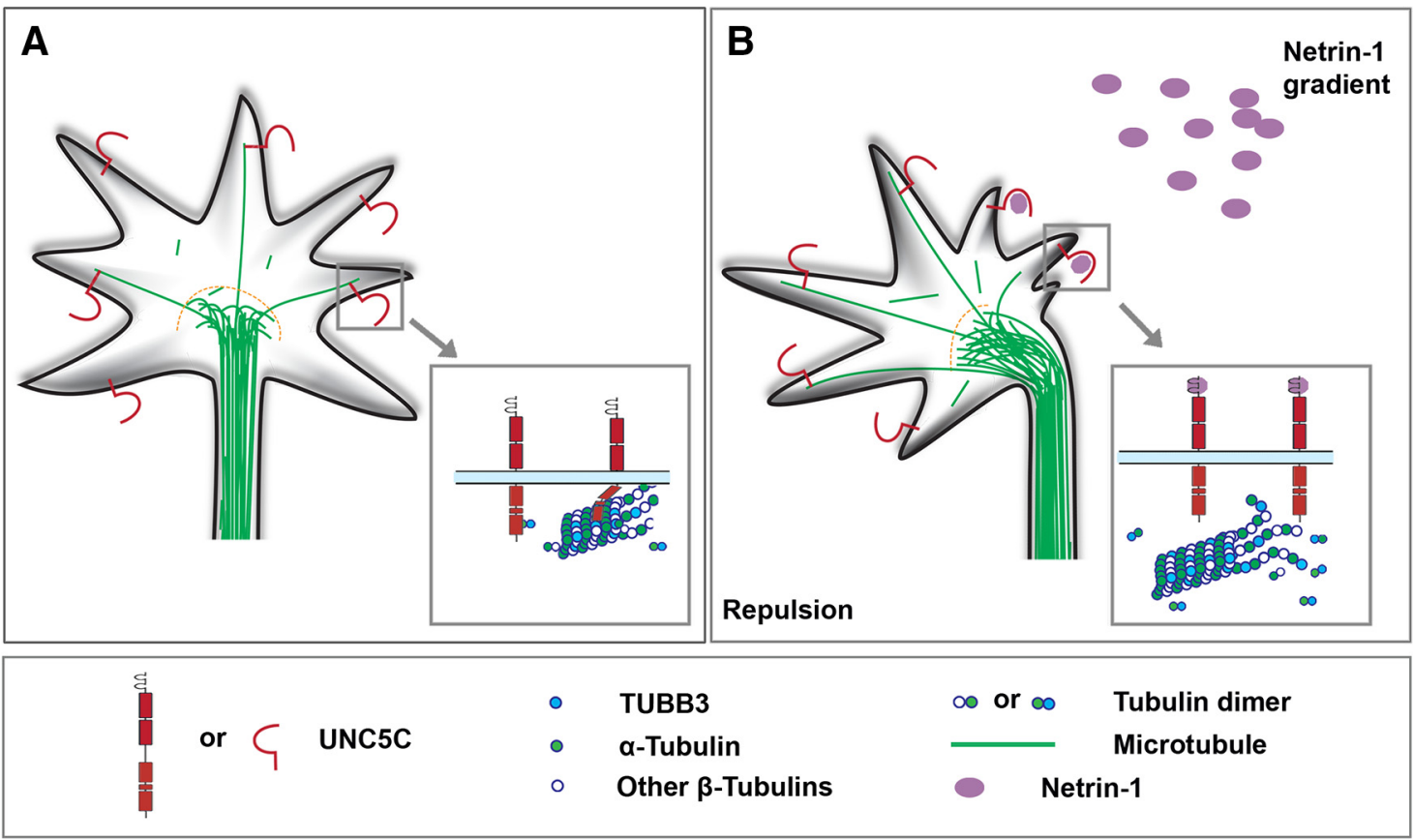

Figure 8. A working model of direct involvement of MT dynamics in netrin-1-mediated axon repulsion. $A, A$ typical $G C$ of developing neurons with lamelliopodia and finger-like filopodia. In the peripheral region of the GC, UNC5C interacts with TUBB3 in "pioneer polymerized MTs" in the absence of netrin-1 gradient. $B$, Binding of netrin- 1 to UNC5C on the side of $\mathrm{GC}$ close to the netrin-1 gradient differentially reduces the interaction of UNC5C with polymerized TUBB3 in MTs and triggers $\mathrm{GC}$ collapse on that side resulting in axon repulsion, despite the fact that netrin-1 induces MT polyermization/dynamics in the GC.

that differential dissociation of UNC5C with polymerized TUBB3 in the GC is required for netrin-1-induced axon repulsion.

\section{The working model of direct modulation of MT dynamics in netrin-1 signaling}

Modulation of MT dynamics plays an instructive role in guiding GC navigation. However, whether MT dynamics are directly regulated by guidance cues is still unclear. Our recent studies have shown that netrin-1 signaling directly regulates MT dynamics through coupling its receptor DCC and DSCAM to TUBB3 in axon outgrowth, branching, and attraction (Qu et al., 2013a; Huang et al., 2015). In this model, netrin-1 may differentially promote MT dynamics and local stabilization of MTs in the proximal region of the GC through the interaction of DCC and/or DSCAM with polymerized TUBB3, which could enable dynamic MTs to differentially protrude into the proximal region and further GC protrusion on the proximal side. In contrast, the distal region of the GC, including lamellipodia and filopodia, would collapse and the GC would eventually turn toward the netrin-1 source. Moreover, our current results provide a working model of netrin-1-mediated axon repulsion (Fig. 8). Netrin-1 differentially induces MT dynamics in the GC with more MT growth in the distal region than the proximal region; at the same time, netrin-1 promotes dissociation of polymerized TUBB3 in MTs with UNC5C in the GC, which may result in less polymerized MTs "captured" by UNC5C on the proximal side than on the distal side of the GC, leading to retraction of lamellipodia and filopodia in the proximal region and axon repulsion. In both models, in response to guidance cues, the "capture" of dynamic MTs by guidance receptors in the GC functions as a molecular switch: coupling of receptors with dynamic MTs could stabilize and promote MT growth in the GC and then trigger axon outgrowth, branching, and attraction; uncoupling of polymerized
MTs with guidance receptors may induce retraction and collapse of the GC and cause axon repulsion.

Previous studies on MT dynamics in axon turning also support our working mode. For instance, dynamic MTs are preferentially oriented and stabilized in the direction of GC turn (Tanaka and Kirschner, 1995); local stabilization of MTs in one side of the GC is sufficient to cause the GC turn to that side (Buck and Zheng, 2002); stabilizing MTs and promoting their polymerization at plus ends increase axon outgrowth in vitro and in vivo (Sengottuvel et al., 2011); asymmetric redistribution of dynamic MTs toward the far side of the GC is involved in Wnt5a-promoted cortical axon outgrowth and repulsion ( $\mathrm{Li}$ et al., 2014).

TUBB3 is a key downstream component in netrin-1 signaling Activation of UNC5 signaling is essential for netrin-1-mediated repulsion. In this study, we found that netrin-1 repelled the GC of primary P2 cerebellar EGL neurons (Fig. 4), and both UNC5C shRNA and TUBB3 shRNA inhibited netrin-1-induced GC repulsion (Fig. 4). Expression of wild-type UNC5C or TUBB3 rescued the effect of UNC5C or TUBB3 knockdown on netrin1-promoted GC repulsion, respectively (Fig. 4). These results indicate that TUBB3 is specifically involved in netrin-1/ $\mathrm{UNC5C}$-induced axon repulsion in vitro. Results from in ovo electroporation studies with chick spinal cords demonstrate that both UNC5C and TUBB3 are essential for netrin-1-mediated DRG axon projection in vivo (Fig. 7). Together, these studies strongly support the idea that TUBB3 is required for netrin-1/ UNC5C-mediated axon repulsion in the developing nervous system. It is worth noting that the dorsal spinal cord is the centrally projecting DRG axon target; and in our in ovo electroporation experiments, knockdown of UNC5C and TUBB3 in this region could also affect the DRG axon projection. To clarify this issue, future investigations will be focused on analyzing DREZ phenotypes after 
knockdown or knock-out of UNC5C and TUBB3 in DRG neurons only.

Mammalian UNC5 has four homologs: UNC5A-UNC5D. UNC5A (Bartoe et al., 2006) and UNC5B (Hata et al., 2009) were reported to mediate netrin-1-induced axon GC collapse of primary hippocampal and cortical neurons, respectively. Although our results indicate that UNC5C specifically interacts with TUBB3, not TUBB1 or TUBB2, it remains to be determined whether other UNC5 homologs, such as UNC5A, UNC5B, and UNC5D, can functionally associate with these $\beta$-tubulin isoforms in netrin-1-mediated repulsion. In response to netrin-1, UNC-5 coordinates with either DCC or DSCAM to initiate axon repulsion (Keino-Masu et al., 1996; Kolodziej et al., 1996; Leonardo et al., 1997; Hong et al., 1999; Finger et al., 2002; Purohit et al., 2012), whereas collaboration of DCC with DSCAM mediates chemoattraction (Ly et al., 2008; Liu et al., 2009; Qu et al., 2013b; Huang et al., 2015). Our recent studies suggest that DSCAM coordinates with DCC in coupling netrin-1 signaling to MT dynamics via dynamic TUBB3 to promote axon outgrowth, branching, and attraction (Qu et al., 2013a; Huang et al., 2015). In future studies, it will be interesting to determine the role of TUBB3 in coordination of UNC5 with DSCAM or DCC in netrin-1 repulsion.

TUBB3 mutations cause a spectrum of defects in axonal projection and neuronal migration, including agenesis or hypoplasia of commissural and cranial axons, misorientation and dysgenesis of the corticospinal tract, basal ganglia dysmorphisms, malformation of cortical development associated with neuronal migration defects, hypoplasia of hippocampi, thalami, and brainstem, and cerebellar dysplasia (Poirier et al., 2010; Tischfield et al., 2010; Whitman et al., 2016). Although the disease-associated TUBB3 mutations can impair tubulin heterodimer formation, disrupt kinesin-MT interactions, and alter MT instability (Poirier et al., 2010; Tischfield et al., 2010; Whitman et al., 2016), how these mutations cause specific defects in axon guidance and neuronal migration is unclear, considering that TUBB3 is widely expressed in all neurons in the developing nervous system. TUBB3 is required for netrin-1-mediated axon attraction and repulsion, suggesting a novel mechanism underlying these mutation-related defects in brain development. Further studies will be necessary to determine whether disease-related TUBB3 mutations affect the interactions with UNC5C, DCC, and DSCAM, impair netrin-1regulated MT dynamics in the GC, and disrupt netrin-1-mediated chemoattraction and chemorepulsion in the developing nervous system.

TUBB1, TUBB2, and TUBB3 are encoded by different genes and are well conserved across species. Amino acid sequences of these $\beta$-tubulins largely differ at the $\mathrm{C}$ terminus, which is the major region associating with multiple MT-associated proteins (Sullivan and Cleveland, 1986). Also, these $\beta$-tubulin isotypes display differential tissue expression patterns: TUBB1 is ubiquitous, whereas TUBB2 and TUBB3 are restricted to neuronal tissues (Sullivan et al., 1986; Wang et al., 1986; Cleveland, 1987; Leandro-García et al., 2010). MT dynamics could be affected by the tubulin isotype composition, and TUBB3 was thought to be more dynamic than other $\beta$-tubulin isotypes (Panda et al., 1994). Mutations of TUBB1, TUBB2, or TUBB3 cause developmental disorders with different phenotypic spectra (Jaglin et al., 2009; Guerrini et al., 2012; Cushion et al., 2013). These results suggest that these $\beta$-tubulin isotypes may be differentially involved in tubulin heterodimer formation and MT stability in developing neurons. The direct interaction of UNC5C with TUBB3, but not TUBB1 or TUBB2, may rely on the difference of their sequences, protein confirmation, expression patterns, and isotype-engaged MT dynamics.

\section{References}

Ackerman SL, Kozak LP, Przyborski SA, Rund LA, Boyer BB, Knowles BB (1997) The mouse rostral cerebellar malformation gene encodes an UNC-5-like protein. Nature 386:838-842. CrossRef Medline

Alcántara S, Ruiz M, De Castro F, Soriano E, Sotelo C (2000) Netrin 1 acts as an attractive or as a repulsive cue for distinct migrating neurons during the development of the cerebellar system. Development 127:1359-1372. Medline

Avraham O, Hadas Y, Vald L, Hong S, Song MR, Klar A (2010) Motor and dorsal root ganglion axons serve as choice points for the ipsilateral turning of dI3 axons. J Neurosci 30:15546-15557. CrossRef Medline

Bartoe JL, McKenna WL, Quan TK, Stafford BK, Moore JA, Xia J, Takamiya K, Huganir RL, Hinck L (2006) Protein interacting with C-kinase 1/ protein kinase Calpha-mediated endocytosis converts netrin-1-mediated repulsion to attraction. J Neurosci 26:3192-3205. CrossRef Medline

Bronner-Fraser M, Fraser S (1989) Developmental potential of avian trunk neural crest cells in situ. Neuron 3:755-766. CrossRef Medline

Buck KB, Zheng JQ (2002) Growth cone turning induced by direct local modification of microtubule dynamics. J Neurosci 22:9358-9367. Medline

Challacombe JF, Snow DM, Letourneau PC (1997) Dynamic microtubule ends are required for growth cone turning to avoid an inhibitory guidance cue. J Neurosci 17:3085-3095. Medline

Cleveland DW (1987) The multitubulin hypothesis revisited: what have we learned? J Cell Biol 104:381-383. CrossRef Medline

Colamarino SA, Tessier-Lavigne M (1995) The axonal chemoattractant netrin-1 is also a chemorepellent for trochlear motor axons. Cell 81:621629. CrossRef Medline

Conde C, Cáceres A (2009) Microtubule assembly, organization and dynamics in axons and dendrites. Nat Rev Neurosci 10:319-332. CrossRef Medline

Cushion TD, Dobyns WB, Mullins JG, Stoodley N, Chung SK, Fry AE, Hehr U, Gunny R, Aylsworth AS, Prabhakar P, Uyanik G, Rankin J, Rees MI, Pilz DT (2013) Overlapping cortical malformations and mutations in TUBB2B and TUBA1A. Brain 136:536-548. CrossRef Medline

Dent EW, Gertler FB (2003) Cytoskeletal dynamics and transport in growth cone motility and axon guidance. Neuron 40:209-227. CrossRef Medline

Dent EW, Barnes AM, Tang F, Kalil K (2004) Netrin-1 and semaphorin $3 \mathrm{~A}$ promote or inhibit cortical axon branching, respectively, by reorganization of the cytoskeleton. J Neurosci 24:3002-3012. CrossRef Medline

Dent EW, Gupton SL, Gertler FB (2011) The growth cone cytoskeleton in axon outgrowth and guidance. Cold Spring Harb Perspect Biol 3:a001800. CrossRef Medline

Dunn KW, Kamocka MM, McDonald JH (2011) A practical guide to evaluating colocalization in biological microscopy. Am J Physiol Cell Physiol 300:C723-C742. CrossRef Medline

Fazeli A, Dickinson SL, Hermiston ML, Tighe RV, Steen RG, Small CG, Stoeckli ET, Keino-Masu K, Masu M, Rayburn H, Simons J, Bronson RT, Gordon JI, Tessier-Lavigne M, Weinberg RA (1997) Phenotype of mice lacking functional Deleted in colorectal cancer (Dcc) gene. Nature 386: 796-804. CrossRef Medline

Finger JH, Bronson RT, Harris B, Johnson K, Przyborski SA, Ackerman SL (2002) The netrin 1 receptors Unc5h3 and Dcc are necessary at multiple choice points for the guidance of corticospinal tract axons. J Neurosci 22:10346-10356. Medline

Guan KL, Rao Y (2003) Signalling mechanisms mediating neuronal responses to guidance cues. Nat Rev Neurosci 4:941-956. CrossRef Medline

Guerrini R, Mei D, Cordelli DM, Pucatti D, Franzoni E, Parrini E (2012) Symmetric polymicrogyria and pachygyria associated with TUBB2B gene mutations. Eur J Hum Genet 20:995-998. CrossRef Medline

Hata K, Kaibuchi K, Inagaki S, Yamashita T (2009) Unc5B associates with LARG to mediate the action of repulsive guidance molecule. J Cell Biol 184:737-750. CrossRef Medline

Hedgecock EM, Culotti JG, Hall DH (1990) The unc-5, unc-6, and unc-40 genes guide circumferential migrations of pioneer axons and mesodermal cells on the epidermis in C. elegans. Neuron 4:61-85. CrossRef Medline 
Hong K, Hinck L, Nishiyama M, Poo MM, Tessier-Lavigne M, Stein E (1999) A ligand-gated association between cytoplasmic domains of UNC5 and DCC family receptors converts netrin-induced growth cone attraction to repulsion. Cell 97:927-941. CrossRef Medline

Horn AE, Kugel JF, Goodrich JA (2016) Single molecule microscopy reveals mechanistic insight into RNA polymerase II preinitiation complex assembly and transcriptional activity. Nucleic Acids Res 44:71327143. CrossRef Medline

Huang H, Shao Q, Qu C, Yang T, Dwyer T, Liu G (2015) Coordinated interaction of Down syndrome cell adhesion molecule and deleted in colorectal cancer with dynamic TUBB3 mediates Netrin-1-induced axon branching. Neuroscience 293:109-122. CrossRef Medline

Ishii N, Wadsworth WG, Stern BD, Culotti JG, Hedgecock EM (1992) UNC-6, a laminin-related protein, guides cell and pioneer axon migrations in C. elegans. Neuron 9:873-881. CrossRef Medline

Jaglin XH, Poirier K, Saillour Y, Buhler E, Tian G, Bahi-Buisson N, FalletBianco C, Phan-Dinh-Tuy F, Kong XP, Bomont P, Castelnau-Ptakhine L, Odent S, Loget P, Kossorotoff M, Snoeck I, Plessis G, Parent P, Beldjord C, Cardoso C, Represa A, et al. (2009) Mutations in the beta-tubulin gene TUBB2B result in asymmetrical polymicrogyria. Nat Genet 41:746-752. CrossRef Medline

Kalil K, Dent EW (2005) Touch and go: guidance cues signal to the growth cone cytoskeleton. Curr Opin Neurobiol 15:521-526. CrossRef Medline

Keino-Masu K, Masu M, Hinck L, Leonardo ED, Chan SS, Culotti JG, TessierLavigne M (1996) Deleted in Colorectal Cancer (DCC) encodes a netrin receptor. Cell 87:175-185. CrossRef Medline

Kennedy TE, Serafini T, de la Torre JR, Tessier-Lavigne M (1994) Netrins are diffusible chemotropic factors for commissural axons in the embryonic spinal cord. Cell 78:425-435. CrossRef Medline

Kennedy TE, Wang H, Marshall W, Tessier-Lavigne M (2006) Axon guidance by diffusible chemoattractants: a gradient of netrin protein in the developing spinal cord. J Neurosci 26:8866-8874. CrossRef Medline

Kolodkin AL, Tessier-Lavigne M (2011) Mechanisms and molecules of neuronal wiring: a primer. Cold Spring Harb Perspect Biol 3:a001727. CrossRef Medline

Kolodziej PA, Timpe LC, Mitchell KJ, Fried SR, Goodman CS, Jan LY, Jan YN (1996) frazzled encodes a Drosophila member of the DCC immunoglobulin subfamily and is required for CNS and motor axon guidance. Cell 87:197-204. CrossRef Medline

Lai Wing Sun K, Correia JP, Kennedy TE (2011) Netrins: versatile extracellular cues with diverse functions. Development 138:2153-2169. CrossRef Medline

Leandro-García LJ, Leskelä S, Landa I, Montero-Conde C, López-Jiménez E, Letón R, Cascón A, Robledo M, Rodríguez-Antona C (2010) Tumoral and tissue-specific expression of the major human beta-tubulin isotypes. Cytoskeleton 67:214-223. CrossRef Medline

Leonardo ED, Hinck L, Masu M, Keino-Masu K, Ackerman SL, TessierLavigne M (1997) Vertebrate homologues of C. elegans UNC-5 are candidate netrin receptors. Nature 386:833-838. CrossRef Medline

Li L, Fothergill T, Hutchins BI, Dent EW, Kalil K (2014) Wnt5a evokes cortical axon outgrowth and repulsive guidance by tau mediated reorganization of dynamic microtubules. Dev Neurobiol 74:797-817. CrossRef Medline

Li X, Gao X, Liu G, Xiong W, Wu J, Rao Y (2008) Netrin signal transduction and the guanine nucleotide exchange factor DOCK180 in attractive signaling. Nat Neurosci 11:28-35. CrossRef Medline

Liu G, Dwyer T (2014) Microtubule dynamics in axon guidance. Neurosci Bull 30:569-583. CrossRef Medline

Liu G, Beggs H, Jürgensen C, Park HT, Tang H, Gorski J, Jones KR, Reichardt LF, Wu J, Rao Y (2004) Netrin requires focal adhesion kinase and Src family kinases for axon outgrowth and attraction. Nat Neurosci 7:12221232. CrossRef Medline

Liu G, Li W, Gao X, Li X, Jürgensen C, Park HT, Shin NY, Yu J, He ML, Hanks SK, Wu JY, Guan KL, Rao Y (2007) p130CAS is required for netrin signaling and commissural axon guidance. J Neurosci 27:957-968. CrossRef Medline

Liu G, Li W, Wang L, Kar A, Guan KL, Rao Y, Wu JY (2009) DSCAM functions as a netrin receptor in commissural axon pathfinding. Proc Natl Acad Sci U S A 106:2951-2956. CrossRef Medline

Lowery LA, Van Vactor D (2009) The trip of the tip: understanding the growth cone machinery. Nat Rev Mol Cell Biol 10:332-343. CrossRef Medline

Ly A, Nikolaev A, Suresh G, Zheng Y, Tessier-Lavigne M, Stein E (2008) DSCAM is a netrin receptor that collaborates with DCC in mediating turning responses to netrin-1. Cell 133:1241-1254. CrossRef Medline

Masuda T, Watanabe K, Sakuma C, Ikenaka K, Ono K, Yaginuma H (2008) Netrin- 1 acts as a repulsive guidance cue for sensory axonal projections toward the spinal cord. J Neurosci 28:10380-10385. CrossRef Medline

Masuda T, Yaginuma H, Sakuma C, Ono K (2009) Netrin-1 signaling for sensory axons: involvement in sensory axonal development and regeneration. Cell Adh Migr 3:171-173. CrossRef Medline

Palmesino E, Haddick PC, Tessier-Lavigne M, Kania A (2012) Genetic analysis of DSCAM's role as a Netrin-1 receptor in vertebrates. J Neurosci 32:411-416. CrossRef Medline

Panda D, Miller HP, Banerjee A, Ludueña RF, Wilson L (1994) Microtubule dynamics in vitro are regulated by the tubulin isotype composition. Proc Natl Acad Sci U S A 91:11358-11362. CrossRef Medline

Poirier K, Saillour Y, Bahi-Buisson N, Jaglin XH, Fallet-Bianco C, Nabbout R, Castelnau-Ptakhine L, Roubertie A, Attie-Bitach T, Desguerre I, Genevieve D, Barnerias C, Keren B, Lebrun N, Boddaert N, Encha-Razavi F, Chelly J (2010) Mutations in the neuronal ss-tubulin subunit TUBB3 result in malformation of cortical development and neuronal migration defects. Hum Mol Genet 19:4462-4473. CrossRef Medline

Purohit AA, Li W, Qu C, Dwyer T, Shao Q, Guan KL, Liu G (2012) Down syndrome cell adhesion molecule (DSCAM) associates with uncoordinated-5C (UNC5C) in netrin-1-mediated growth cone collapse. J Biol Chem 287: 27126-27138. CrossRef Medline

Qu C, Dwyer T, Shao Q, Yang T, Huang H, Liu G (2013a) Direct binding of TUBB3 with DCC couples netrin-1 signaling to intracellular microtubule dynamics in axon outgrowth and guidance. J Cell Sci 126:3070-3081. CrossRef Medline

Qu C, Li W, Shao Q, Dwyer T, Huang H, Yang T, Liu G (2013b) c-Jun $\mathrm{N}$-terminal kinase 1 (JNK1) is required for coordination of netrin signaling in axon guidance. J Biol Chem 288:1883-1895. CrossRef Medline

Quinn CC, Wadsworth WG (2008) Axon guidance: asymmetric signaling orients polarized outgrowth. Trends Cell Biol 18:597-603. CrossRef Medline

Ruffins S, Artinger KB, Bronner-Fraser M (1998) Early migrating neural crest cells can form ventral neural tube derivatives when challenged by transplantation. Dev Biol 203:295-304. CrossRef Medline

Sengottuvel V, Leibinger M, Pfreimer M, Andreadaki A, Fischer D (2011) Taxol facilitates axon regeneration in the mature CNS. J Neurosci 31: 2688-2699. CrossRef Medline

Serbedzija GN, Bronner-Fraser M, Fraser SE (1989) A vital dye analysis of the timing and pathways of avian trunk neural crest cell migration. Development 106:809-816. Medline

Sullivan KF, Cleveland DW (1986) Identification of conserved isotypedefining variable region sequences for four vertebrate beta tubulin polypeptide classes. Proc Natl Acad Sci U S A 83:4327-4331. CrossRef Medline

Sullivan KF, Machlin PS, Ratrie H 3rd, Cleveland DW (1986) Sequence and expression of the chicken beta 3 tubulin gene: a vertebrate testis betatubulin isotype. J Biol Chem 261 13317-13322.

Suter DM, Forscher P (2000) Substrate-cytoskeletal coupling as a mechanism for the regulation of growth cone motility and guidance. J Neurobiol 44:97-113. CrossRef Medline

Tanaka E, Kirschner MW (1995) The role of microtubules in growth cone turning at substrate boundaries. J Cell Biol 128:127-137. CrossRef Medline

Tanaka E, Sabry J (1995) Making the connection: cytoskeletal rearrangements during growth cone guidance. Cell 83:171-176. CrossRef Medline

Tcherkezian J, Brittis PA, Thomas F, Roux PP, Flanagan JG (2010) Transmembrane receptor DCC associates with protein synthesis machinery and regulates translation. Cell 141:632-644. CrossRef Medline

Tessier-Lavigne M, Placzek M, Lumsden AG, Dodd J, Jessell TM (1988) Chemotropic guidance of developing axons in the mammalian central nervous system. Nature 336:775-778. CrossRef Medline

Tischfield MA, Baris HN, Wu C, Rudolph G, Van Maldergem L, He W, Chan WM, Andrews C, Demer JL, Robertson RL, Mackey DA, Ruddle JB, Bird 
TD, Gottlob I, Pieh C, Traboulsi EI, Pomeroy SL, Hunter DG, Soul JS, Newlin A, et al. (2010) Human TUBB3 mutations perturb microtubule dynamics, kinesin interactions, and axon guidance. Cell 140:74-87. CrossRef Medline

Vitriol EA, Zheng JQ (2012) Growth cone travel in space and time: the cellular ensemble of cytoskeleton, adhesion, and membrane. Neuron 73: 1068-1081. CrossRef Medline

Wang D, Villasante A, Lewis SA, Cowan NJ (1986) The mammalian betatubulin repertoire: hematopoietic expression of a novel, heterologous beta-tubulin isotype. J Cell Biol 103:1903-1910. CrossRef Medline

Watanabe K, Tamamaki N, Furuta T, Ackerman SL, Ikenaka K, Ono K (2006) Dorsally derived netrin 1 provides an inhibitory cue and elaborates the 'waiting period' for primary sensory axons in the developing spinal cord. Development 133:1379-1387. CrossRef Medline

Whitman MC, Andrews C, Chan WM, Tischfield MA, Stasheff SF, Brancati F, Ortiz-Gonzalez X, Nuovo S, Garaci F, MacKinnon SE, Hunter DG, Grant PE, Engle EC (2016) Two unique TUBB3 mutations cause both CFEOM3 and malformations of cortical development. Am J Med Genet A 170A:297305. Medline

Yam PT, Langlois SD, Morin S, Charron F (2009) Sonic hedgehog guides axons through a noncanonical, Src-family-kinase-dependent signaling pathway. Neuron 62:349-362. CrossRef Medline

Yu JY, DeRuiter SL, Turner DL (2002) RNA interference by expression of short-interfering RNAs and hairpin RNAs in mammalian cells. Proc Natl Acad Sci U S A 99:6047-6052. CrossRef Medline 